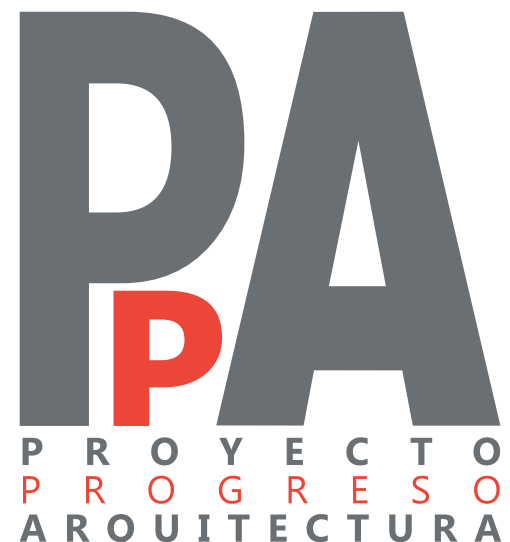

ARQUITECTURAS AMPLIADAS

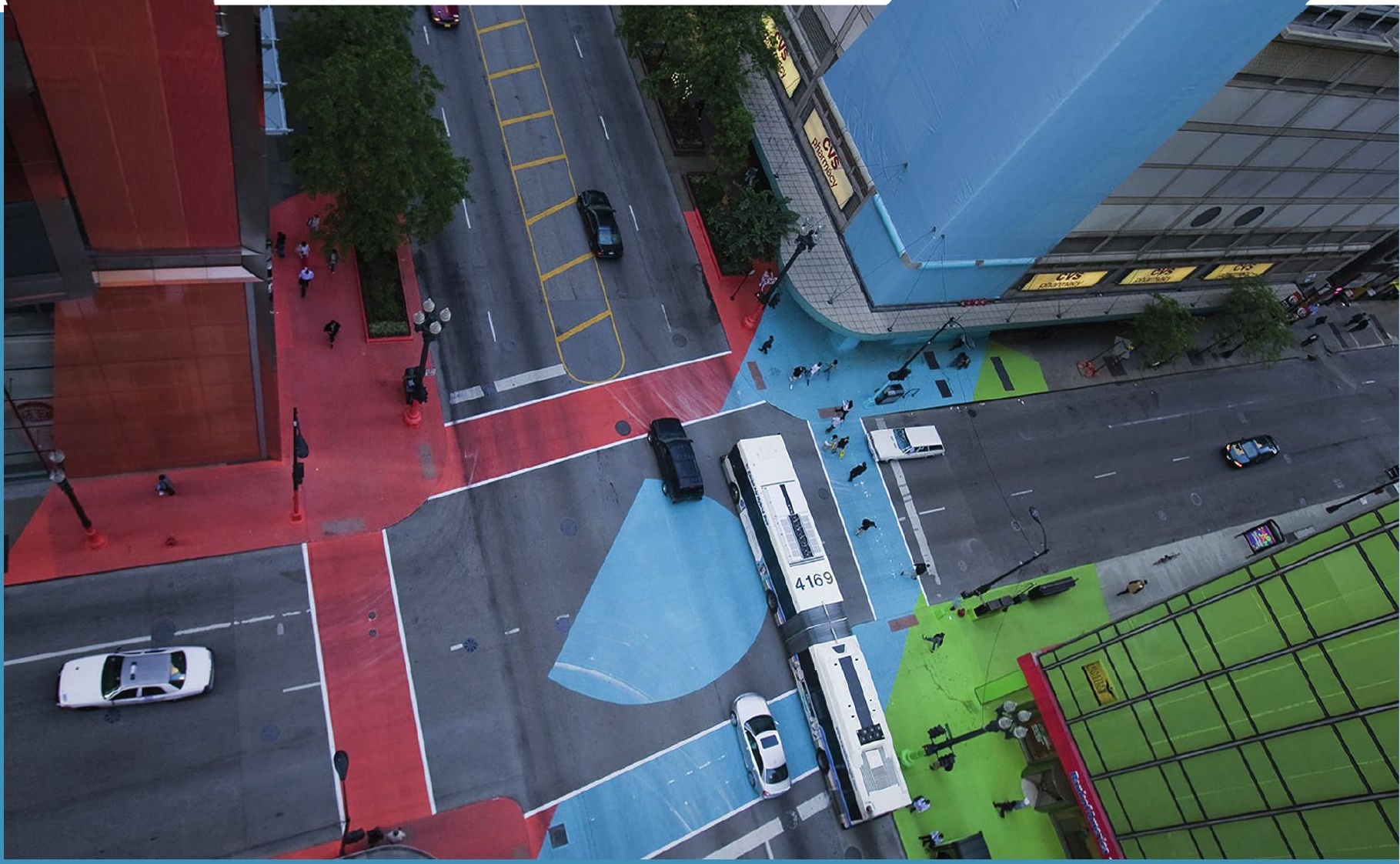




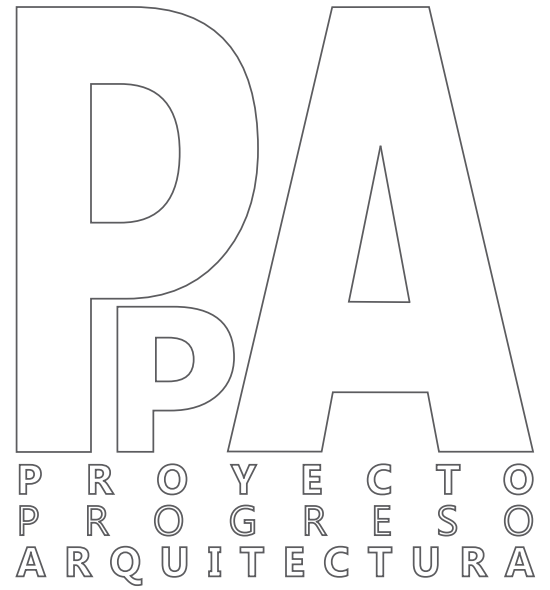

\section{ARQUITECTURAS AMPLIADA}

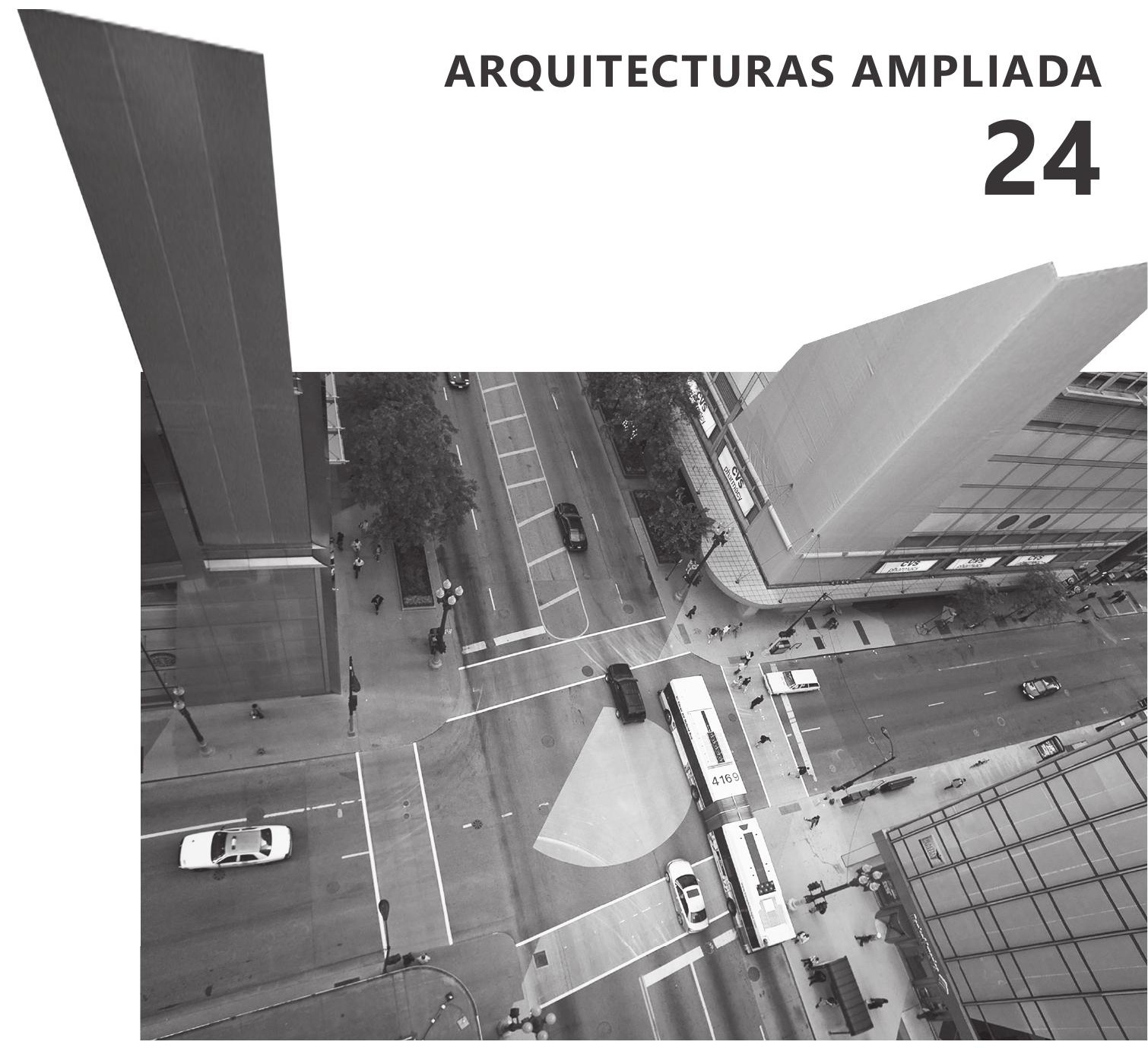

EDITORIAL UNIVERSIDAD DE SEVILLA AÑO 2019. ISSN 2171-6897 ISSNe 2173-1616 DOI: http://dx.doi.org/10.12795/ppa 


\section{REVISTA PROYECTO PROGRESO ARQUITECTURA}

N2/4

arquitecturas ampliadas
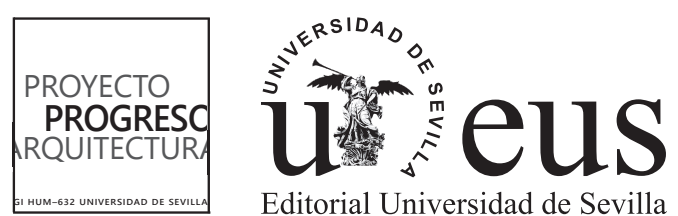


\section{arquitecturas ampliadas}

EDITA

Editorial Universidad de Sevilla. Sevilla

DIRECCIÓN CORRESPONDENCIA CIENTÍFICA

E.T.S. de Arquitectura. Avda Reina Mercedes, nº $241012-$

Sevilla.

Amadeo Ramos Carranza, Dpto. Proyectos Arquitectónicos.

e-mail: revistappa.direccion@gmail.com

\section{EDICIÓN ON-LINE}

Portal informático https://revistascientificas.us.es/index.php/ppa Portal informático Grupo de Investigación HUM-632

http://www. proyectoprogresoarquitectura.com

Portal informático Editorial Universidad de Sevilla http://www. editorial.us.es/

(c) EDITORIAL UNIVERSIDAD DE SEVILLA, 2019.

Calle Porvenir, 27. 41013 SEVILLA. Tfs. 954487447 / 954487451

Fax 954487443. [eus4@us.es] [http://www.editorial.us.es]

(c) TEXTOS: SUS AUTORES,

(C) IMÁGENES: SUS AUTORES Y/O INSTITUCIONES

DISEÑO PORTADA:

Rosa María Añón Abajas - Amadeo Ramos Carranza

En base a la fotografía: (C) Jessica Stockholder, cortesía de la artista y de Mitchell-Innes \& Nash, Nueva York

\section{DISEÑO PLANTILLA PORTADA-CONTRAPORTADA}

Miguel Ángel de la Cova Morillo-Velarde

DISEÑO PLANTILLA MAQUETACIÓN

Maripi Rodríguez

MAQUETACIÓN

Referencias Cruzadas

CORRECCION ORTOTIPOGRÁFICA

José Antonio Duarte

ISSN (ed. impresa): 2171-6897

ISSN-e (ed. electrónica): 2173-1616

DOI: http://dx.doi.org/10.12795/ppa

DEPÓSITO LEGAL: SE-2773-2010

PERIOCIDAD DE LA REVISTA: MAYO Y NOVIEMBRE

IMPRIME: PODIPRINT

Reservados todos los derechos. Ni la totalidad ni parte de esta revista puede reproducirse o transmitirse por ningún procedimiento electrónico o mecánico, incluyendo fotocopia, grabación magnética o cualquier almacenamiento de información y sistema de recuperación, sin permiso escrito de la Editorial Universidad de Sevilla.

Las opiniones y los criterios vertidos por los autores en los artículos firmados son responsabilidad exclusiva de los mismos. 


\section{DIRECCIÓN}

Dr. Amadeo Ramos Carranza. Escuela Técnica Superior de Arquitectura. Universidad de Sevilla. España

\section{SECRETARÍA}

Dra. Rosa María Añón Abajas. Escuela Técnica Superior de Arquitectura. Universidad de Sevilla. España

\section{EQUIPO EDITORIAL \\ Edición:}

Dr. Amadeo Ramos Carranza. Escuela Técnica Superior de Arquitectura. Universidad de Sevilla. España.

Dra. Rosa María Añón Abajas. Escuela Técnica Superior de Arquitectura. Universidad de Sevilla. España.

Dr. Francisco Javier Montero Fernández. Escuela Técnica Superior de Arquitectura. Universidad de Sevilla. España.

Dra. Esther Mayoral Campa. Escuela Técnica Superior de Arquitectura. Universidad de Sevilla. España.

Dr. Miguel Ángel de la Cova Morillo-Velarde. Escuela Técnica Superior de Arquitectura. Universidad de Sevilla. España.

Dr. Germán López Mena. Escuela Técnica Superior de Arquitectura. Universidad de Sevilla. España.

Dra. Gloria Rivero Lamela. Escuela Técnica Superior de Arquitectura. Universidad de Sevilla. España.

Juan José López de la Cruz. Escuela Técnica Superior de Arquitectura. Universidad de Sevilla. España.

Guillermo Pavón Torrejón. Escuela Técnica Superior de Arquitectura. Universidad de Sevilla. España.

Externos edición (asesores):

Dr. José Altés Bustelo. Escuela Técnica Superior de Arquitectura. Universidad de Valladolid. España.

Dr. Carlos Arturo Bell Lemus. Facultad de Arquitectura. Universidad del Atlántico. Colombia.

Dr. José de Coca Leicher. Escuela Técnica Superior de Arquitectura. Universidad Politécnica de Madrid. España. Dra. Patricia de Diego Ruiz. Escuela Técnica Supeiror de Arquitectura y Geodesia. Universidad Alcalá de Heranes. España.

Dr. Alfonso del Pozo y Barajas. Escuela Técnica Superior de Arquitectura. Universidad de Sevilla. España.

Dr. Jaume J. Ferrer Fores. Escola Tècnica Superior

d'Arquitectura de Barcelona. Universitat Politècnica de Catalunya. España.

Dra. Laura MArtínez Guereñu. El School of Architecture \& Design, IE University, Madrid; Segovia. España.

Dra. Clara Mejía Vallejo. Escuela Técnica Superior de Arquitectura. Universidad Politécnica de Valencia. España.

Dra. Luz Paz Agras. Escuela Técnica Superior de Arquitectura. Universidade da Coruña. España.

Dra. Marta Sequeira. CIAUD, Faculdade de Arquitectura da Universidade de Lisboa, Portugal.

\section{SECRETARÍA TÉCNICA}

Dra. Gloria Rivero Lamela. Escuela Técnica Superior de Arquitectura. Universidad de Sevilla. España.

\section{EDITORA Y COORDINACION CONTENIDOS CIENTÍFICOS DEL NÚMERO}

Dra. Rosa María Añón Abajas. Escuela Técnica Superior de Arquitectura. Universidad de Sevilla. España.

\section{COMITÉ CIÉNTIFICO}

Dr. Carlo Azteni. DICAAR. Dipartimento di Ingegneria Civile, Ambientale e Architettura. University Of Cagliari. Italia.

Dra. Maristella Casciato. GETTY Research Institute, GETTY, Los Angeles. Estados Unidos.

Dra. Anne Marie Châtelet. École Nationale Supérieure D'Architecture de Strasbourg (ENSAS). Francia.

Dr. Jean Louis Cohen. Institute of Fine Arts, New York University. Estados Unidos.

Dra. Josefina González Cubero. Escuela Técnica Superior de Arquitectura. Universidad de Valladolid. España.

Dr. José Manuel López Peláez. Escuela Técnica Superior de Arquitectura. Universidad Politécnica de Madrid. España.

Dra. Maite Méndez Baiges. Departamento de Historia del Arte. Universidad de Málaga. España.

Dr. Dietrich C. Neumann. Brown University In Providence, Ri (John Nicholas Brown Center For Public Humanities And Cultural Heritage). Estados Unidos.

Dr. Víctor Pérez Escolano. Catedrático Historia, Teoría y Composición Arquitectónicas. Escuela Técnica Superior de Arquitectura. Universidad de Sevilla. España.

Dr. Jorge Torres Cueco. Catedrático Proyectos Arquitectónicos. Escuela Técnica Superior de Arquitectura. Universitat Politècnica de València. España.

Dr. ir. Frank van der Hoeven, TU DELFT. Architecture and the Built Environment, Netherlands

\section{CORRESPONSALES}

Pablo de Sola Montiel. The Berlage Centre for Advanced Studies in Architecture and Urban Design. Paises Bajos.

Dr. Plácido González Martínez. Tongji University Caup (College Of architectura \& Urban Planing). Shangai, China.

Patrícia Marins Farias. Faculdade de Arquitetura. Universidade Federal da Bahia. Brasil.

Dr. Daniel Movilla Vega. Umeå School of Architecture. Umeå University. Suecia.

Dr. Pablo Sendra Fernández. The Bartlett School of Planning. University College London. Inglaterra.

Alba Zarza Arribas. Centro de Estudos Arnaldo Araújo, Porto. Portugal.

Dra. María Elena Torres Pérez. Facultad de Arquitectura. Universidad Autónoma de Yucatán, Mérida. México.

\section{TEXTOS VIVOS}

Juan José López de la Cruz. Escuela Técnica Superior de Arquitectura. Universidad de Sevilla. España.

Dr. Francisco Javier Montero Fernández. Escuela Técnica Superior de Arquitectura. Universidad de Sevilla. España. Dra. Esther Mayoral Campa. Escuela Técnica Superior de Arquitectura. Universidad de Sevilla. España. 


\section{SERVICIOS DE INFORMACIÓN}

\section{CALIDAD EDITORIAL}

La Editorial Universidad de Sevilla cumple los criterios establecidos por la Comisión Nacional Evaluadora de la Actividad Investigadora para que lo publicado por el mismo sea reconocido como "de impacto" (Ministerio de Ciencia e Innovación, Resolución 18939 de 11 de noviembre de 2008 de la Presidencia de la CNEAI, Apéndice I, BOE n² 282, de 22.11.08).

La Editorial Universidad de Sevilla forma parte de la U.N.E. (Unión de Editoriales Universitarias Españolas) ajustándose al sistema de control de calidad que garantiza el prestigio e internacionalidad de sus publicaciones.

PUBLICATION QUALITY

The Editorial Universidad de Sevilla fulfils the criteria established by the National Commission for the Evaluation of Research Activity (CNEAI) so that its publications are recognised as "of impact" (Ministry of Science and Innovation, Resolution 18939 of 11 November 2008 on the Presidency of the CNEAl, Appendix I, BOE No 282, of 22.11.08).

The Editorial Universidad de Sevilla operates a quality control system which ensures the prestige and international nature of its publications, and is a member of the U.N.E. (Unión de Editoriales Universitarias Españolas-Union of Spanish University Publishers).

Los contenidos de la revista PROYECTO, PROGRESO, ARQUITECTURA aparecen en:

bases de datos: indexación
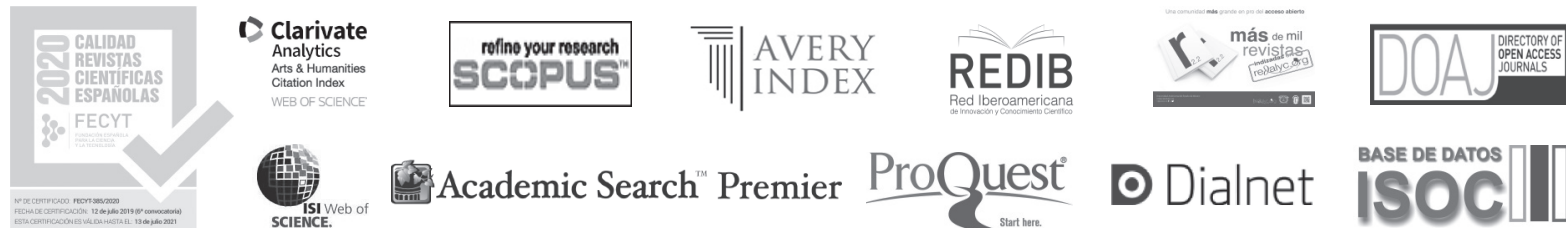

D Dialnet

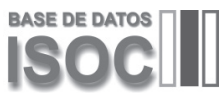

SELLO DE CALIDAD EDITORIAL FECYT 2019. RENOVADO 2020. (Cuartil C3)

WoS. Arts \& Humanities Citation Index

WoS. ESCl - Emerging Sources Citation Index

SCOPUS

AVERY. Avery Index to Architectural Periodicals

REBID. Red Iberoamericana de Innovación y Conocimiento Científico

REDALYC. Red de Revistas Científicas de América Latina y el Caribe, España y Portugal.

EBSCO. Fuente Académica Premier

EBSCO. Art Source

DOAJ, Directory of Open Access Journals

PROQUEST (Arts \& Humanities, full text)

DIALNET

ISOC (Producida por el CCHS del CSIC)

DRIJ. Directory of Research Journals Indexing

SJR (2019): 0.100, H index: 2

\section{catalogaciones: criterios de calidad}

RESH (Revistas Españolas de Ciencias Sociales y Humanidades).

Catálogos CNEAI (16 criterios de 19). ANECA (18 criterios de 21). LATINDEX (35 criterios sobre 36).

DICE (CCHS del CSIC, ANECA).

MIAR, Matriu d'Informació per a l'Avaluació de Revistes. IDCS 2018: 10,500. Campo ARQUITECTURA

CLASIFICACIÓN INTEGRADA DE REVISTAS CIENTÍFICAS (CIRC-CSIC): A

ERIHPLUS

SCIRUS, for Scientific Information.

ULRICH'S WEB, Global Serials Directory.

ACTUALIDAD IBEROAMERICANA.

\section{catálogos on-line bibliotecas notables de arquitectura:}

CLIO. Catálogo on-line. Columbia University. New York

HOLLIS. Catálogo on-line. Harvard University. Cambridge. MA

SBD. Sistema Bibliotecario e Documentale. Instituto Universitario di Architettura di Venezia

OPAC. Servizi Bibliotecari di Ateneo. Biblioteca Centrale. Politecnico di Milano

COPAC. Catálogo colectivo (Reino Unido)

SUDOC. Catálogo colectivo (Francia)

ZBD. Catálogo colectivo (Alemania)

REBIUN. Catálogo colectivo (España)

OCLC. WorldCat (Mundial) 
EVALUACIÓN EXTERNA POR PARES Y ANÓNIMA.

El Consejo Editorial remitirá el artículo a dos expertos revisores anónimos dentro del campo específico de investigación y crítica de arquitectura, según el modelo doble ciego.

El director de la revista comunicará a los autores el resultado motivado de la evaluación por correo electrónico, en la dirección que éstos hayan utilizado para enviar el artículo. El director comunicará al autor principal el resultado de la revisión (publicación sin cambios; publicación con correcciones menores; publicación con correcciones importantes; no aconsejable para su publicación), así como las observaciones y comentarios de los revisores.

Si el manuscrito ha sido aceptado con modificaciones, los autores deberán reenviar una nueva versión del artículo, atendiendo a las demandas y sugerencias de los evaluadores externos. Los artículos con correcciones importantes serán remitidos al Consejo Asesor para verificar la validez de las modificaciones efectuadas por el autor. Los autores pueden aportar también una carta al Consejo Editorial en la que indicarán el contenido de las modificaciones del artículo. Los artículos con correcciones importantes serán remitidos al Consejo Asesor para verificar la validez de las modificaciones efectuadas por el autor.

\section{DECLARACIÓN ÉTICA SOBRE PUBLICACIÓN Y MALAS PRÁCTICAS}

La revista PROYECTO, PROGRESO, ARQUITECTURA (PPA) está comprometida con la comunidad académica en garantizar la ética y calidad de los artículos publicados. Nuestra revista tiene como referencia el Código de Conducta y Buenas Prácticas que, para editores de revistas científicas, define el COMITÉ DE ÉTICA DE PUBLICACIONES (COPE).

Así nuestra revista garantiza la adecuada respuesta a las necesidades de los lectores y autores, asegurando la calidad de lo publicado, protegiendo y respetando el contenido de los artículos y la integridad de los mismo. El Consejo Editorial se compromete a publicar las correcciones, aclaraciones, retracciones y disculpas cuando sea preciso.

En cumplimiento de estas buenas prácticas, la revista PPA tiene publicado el sistema de arbitraje que sigue para la selección de artículos así como los criterios de evaluación que deben aplicar los evaluadores externos -anónimos y por pares, ajenos al Consejo Editorial-. La revista PPA mantiene actualizados estos criterios, basados exclusivamente en la relevancia científica del artículo, originalidad, claridad y pertinencia del trabajo presentado.

Nuestra revista garantiza en todo momento la condifencialidad del proceso de evaluación: el anonimato de los evaluadores y de los autores; el contenido evaluado; los informes razonados emitidos por los evaluadores y cualquier otra comunicación emitida por los consejos Editorial, Asesor y Científico si así procediese.

Igualmente quedan afectados de la máxima confidencialidad las posibles aclaraciones, reclamaciones o quejas que un autor desee remitir a los comités de la revista o a los evaluadores del artículo.

La revista PROYECTO, PROGRESO, ARQUITECTURA (PPA) declara su compromiso por el respeto e integridad de los trabajos ya publicados. Por esta razón, el plagio está estrictamente prohibido y los textos que se identifiquen como plagio o su contenido sea fraudulento, serán eliminados o no publicados por la revista PPA. La revista actuará en estos casos con la mayor celeridad posible. Al aceptar los términos y acuerdos expresados por nuestra revista, los autores han de garantizar que el artículo y los materiales asociados a él son originales o no infringen derechos de autor. También los autores tienen que justificar que, en caso de una autoría compartida, hubo un consenso pleno de todos los autores afectados y que no ha sido presentado ni publicado con anterioridad en otro medio de difusión.

\section{EXTERNAL ANONYMOUS PEER REVIEW.}

Editorial Board will be sent to two anonymous experts, within the specific field of architectural investigation and critique, for a double blind review.

The Director of the journal will communicate the result of the reviewers evaluations to the authors by electronic mail, to the address used to send the article. The Director will communicate the result of the review (publication without changes; publication with minor corrections; publication with significant corrections; its publication is not advisable), as well as the observations and comments of the reviewers, to the main author.

If the manuscript has been accepted with modifications, the authors will have to resubmit a new version of the article, addressing the requirements and suggestions of the external reviewers. The articles with corrections will be sent to Advisory Board for verification of the validity of the modifications made by the author. The authors can also send a letter to the Editorial Board, in which they will indicate the content of the modifications of the article.

\section{ETHICS STATEMENT ON PUBLICATION AND BAD PRACTICES} PROYECTO, PROGRESO ARQUITECTURA (PPA) makes a commitment to the academic community by ensuring the ethics and quality of its published articles. As a benchmark, our journal uses the Code of Conduct and Good Practices which, for scientific journals, is defined for editors by the PUBLICATION ETHICS COMMITTEE (COPE).

Our journal thereby guarantees an appropriate response to the needs of readers and authors, ensuring the quality of the published work, protecting and respecting the content and integrity of the articles. The Editorial Board will publish corrections, clarifications, retractions and apologies when necessary.

In compliance with these best practices, PPA has published the arbitration system that is followed for the selection of articles as well as the evaluation criteria to be applied by the anonymous, external peer-reviewers. PPA keeps these criteria current, based solely on the scientific importance, the originality, clarity and relevance of the presented article.

Our journal guarantees the confidentiality of the evaluation process at all times: the anonymity of the reviewers and authors; the reviewed content; the reasoned report issued by the reviewers and any other communication issued by the editorial, advisory and scientific boards as required.

Equally, the strictest confidentiality applies to possible clarifications, claims or complaints that an author may wish to refer to the journal's committees or the article reviewers.

PROYECTO, PROGRESO ARQUITECTURA (PPA) declares its commitment to the respect and integrity of work already published. For this reason, plagiarism is strictly prohibited and texts that are identified as being plagiarized, or having fraudulent content, will be eliminated or not published in PPA. The journal will act as quickly as possible in such cases. In accepting the terms and conditions expressed by our journal, authors must guarantee that the article and the materials associated with it are original and do not infringe copyright. The authors will also have to warrant that, in the case of joint authorship, there has been full consensus of all authors concerned and that the article has not been submitted to, or previously published in, any other media. 
EVALUADORES EXTERNOS (publicación cada cuatro números, dos años). NÚMEROS 21 a 24 (incluidos)

Álvarez Álvarez, Darío. Catedrático de Universidad / Departamento de Teoría de la Arquitectura y Proyectos Arquitectónicos / ETS Arquitectura / Universidad de Valladolid / España.

Arrieta Berdasco, Valentín. Doctor Arquitecto, Profesor Asociado / Departamento de Teoría de la Arquitectura y Proyectos Arquitectónicos / ETS Arquitectura / Universidad de Valladolid / España.

Bardí i Milá, Berta. Profesora Asociada doctora / / Departamento de Projectes Arquitectònics / ETS Arquitectura / Universitat Politècnica de Catalunya, Barcelona Tech / España.

Bergera Serrano, Iñaki. Titular de Universidad / Unidad Predepartamental de Arquitectura / Área Proyectos Arquitectónicos / El y Arquitectura / Universidad de Zaragoza / España.

Bobbink, Inge. Dr. ir. Architecture / Section of landscape architecture / TU Delft / Países Bajos.

Burriel Bielza, Luis. Profesor Titular / École Nationale Supérieure d'Architecture de Paris-Belleville / Francia

Calatrava Escobar, Juan. Catedrático de Universidad / Departamento de Construcciones Arquitectónicas / ETS Arquitectura / Universidad de Granada / España.

Castellanos Gómez, Raúl. Titular de Universidad / Departamento de Proyectos Arquitectónicos / ETS Arquitectura / Universidad Politécnica de Valencia / España.

Centellas Soler, Miguel. Titular de Universidad / Departamento de Arquitectura y Tecnología de la Edificación / ETS Arquitectura y Edificación / Universidad Politécnica de Cartagena / España.

Chías Navarro, Pilar. Catedrática de Universidad / Departamento de Arquitectura / ETS Arquitectura y Geodesia / Universidad de Alcalá de Henares / España.

de Diego Ruiz, Patricia. Doctora arquitecta, Profesora Asociada / Departamento de Arquitectura / ETS Arquitectura y Geodesia / Universidad de Alcalá de Henares / España.

de la Iglesia Salgado, Félix. Profesor Contratado Doctor / Departamento de Proyectos Arquitectónicos / ETS Arquitectura / Universidad de Sevilla / España.

de la O Cabrera, Manuel Rodrigo. Doctor Arquitecto, Profesor Asociado / Departamento de Composición Arquitectónica / ETS Arquitectura / Universidad Politécnica de Madrid / España.

Delgado Orusco, Eduardo. Profesor Ayudante Doctor / Departamento de Arquitectura. Área de Proyectos Arquitectónicos / Escuela de Ingeniería y Arquitectura / Universidad de Zaragoza / España.

Deltell Pastor, Juan. Titular de Universidad / Departamento de Proyectos Arquitectónicos / ETS Arquitectura / Universitat Politècnica de València / España.

Diañez Rubio, Pablo. Titular de Universidad / Departamento de Proyectos Arquitectónicos / ETS Arquitectura / Universidad de Sevilla / España.

Díaz Segura, Alfonso. Titular de Universidad / Proyectos, Teoría y Técnica del Diseño y la Arquitectura / ETS Arquitectura / Universidad CEU Cardenal Herrera, Valencia / España.

Domingo Calabuig, Débora. Titular de Universidad / Departamento de Proyectos Arquitectónicos / ETS Arquitectura / Universidad Politécnica de Valencia / España.

Fernández Fariña, Almudena. Profesora Contratada Doctora / Departamento de Pintura / Facultad de Bellas Artes / Universidad de Vigo / España.

Fernández-Trapa de Isasi, Justo. Catedrático de Universidad / Departamento de Proyectos Arquitectónicos / ETS Arquitectura / Universidad Politécnica de Madrid / España.

García Escudero, Daniel. Dr. Arquitecto, Profesor Lector / Departamento de Projectes Arquitectònics / ETS Arquitectura / Universitat Politècnica de Catalunya, Barcelona Tech / España.

González Cubero, Josefina. Titular de Universidad / Departamento de Teoría de la Arquitectura y Proyectos Arquitectónicos / ETS Arquitectura / Universidad de Valladolid / España.

González Fraile, Eduardo. Catedrático de Universidad / Departamento de Teoría de la Arquitectura y Proyectos Arquitectónicos / ETS Arquitectura / Universidad de Valladolid / España.

Gorostiza López, Jorge. Doctor Arquitecto. Cineasta / España.

Hernández Moreno, Silverio. Profesor investigador titular / Facultad de Arquitectura y Diseño / Universidad Autónoma del Estado de México / México.

Labarta Aizpún, Carlos. Titular de Universidad / Unidad Predepartamental de Arquitectura / Área Proyectos Arquitectónicos / Escuela de Ingeniería y Arquitectura / Universidad de Zaragoza / España.

Lizondo Sevilla, Laura. Titular de Universidad / Departamento de Proyectos Arquitectónicos / ETS Arquitectura / Universidad Politécnica de Valencia / España.

Llopis Verdú, Jorge. Catedrático de Universidad / Departamento de Expresión Gráfica Arquitectónica / ETS Arquitectura / Universidad Politécnica de Valencia / España.

López Bahut, Emma. Profesora Contratada Doctora / Departamento de Proyectos arquitectónicos, Urbanismo y Composición / ETS Arquitectura / Universidade da Coruña / España.

López Fernández, Andrés. Titular de Universidad / Departamento de Proyectos Arquitectónicos / ETS Arquitectura / Universidad de Sevilla / España.

Loren Méndez, Mar. Catedrática de Universidad / Departamento de Historia, Teoría y Composición Arquitectónica / ETS Arquitectura / Universidad de Sevilla / España.

Maino Ansaldo, Sandro. Doctor Arquitecto / Departamento Arquitectura / Universidad Técnica Federico Santa María / Chile.

Mària i Serrano, Magda. Profesora Contratada Doctor / Departamento de Projectes Arquitectònics / ETS Arquitectura del Vallès / Universitat Politècnica de Catalunya / España

Marson, Anna. Profesora Ordinario / Dipartimento di culture del progetto / Istituto Universitario di Architettura di Venezia / Università di Venezia / Italia.

Martínez Díaz, Ángel. Titular de Universidad / Departamento de Ideación Gráfica Arquitectónica / ETS Arquitectura / Universidad Politécnica de Madrid / España. 
Mejía Vallejo, Clara. Titular de Universidad / Departamento de Proyectos Arquitectónicos / ETS Arquitectura / Universidad Politécnica de Valencia / España.

Mercader Moyano, Pilar. Titular de Universidad / Departamento de Proyectos Arquitectónicos / ETS Arquitectura / Universidad de Sevilla / España.

Mercé Hospital, José María. Catedrático de Universidad / Departamento de Proyectos Arquitectónicos / ETS Arquitectura y Geodesia / Universidad Alcalá de Henares / España.

Merí de la Maza, Ricardo. Titular de Universidad / Departamento de Proyectos Arquitectónicos / ETS Arquitectura / Universidad Politécnica de Valencia / España.

Mestre Martínez, Nieves. Doctora arquitecta, Profesora Asociada / Departamento de Proyectos Arquitectónicos / ETS Arquitectura / Universidad Politécnica de Madrid / España.

Millán Gómez, Antonio. Catedrático de Universidad / Departamento d’Expressió Gràfica Arquitectònica I / ETS Arquitectura del Vallès / Universitat Politècnica de Catalunya / España.

Moreno Pérez, José Ramón. Titular de Universidad / Dpto. Historia, Teoría y Composición Arquitectónica / ETS Arquitectura / Universidad de Sevilla / España.

Nijhuis, Steffen. Associate Professor. Head of Landscape Architecture Research / Section of Landscape Architecture / Department of Urbanism / Faculty of Architecture and the Built Environment / TU Delft / Países Bajos.

Ojeda Rivera, Juan Francisco. Catedrático de Universidad / Departamento de Geografía, Historia y Filosofía / Universidad Pablo de Olavide, Sevilla / España.

Paz-Agras, Luz. Profesora Ayudante doctor / Departamento de Proyectos Arquitectónicos, Urbanismo y Composición. Área de Composición Arquitectónica / ETS Arquitectura / Universidad de A Coruña / España

Pérez Moreno, Lucía C. Titular de Universidad / Departamento de Arquitectura. Área de Composición Arquitectónica / Escuela de Ingeniería y Arquitectura / Universidad de Zaragoza / España.

Pina Lupiañez, Rafael. Profesor Contratado Doctor / Departamento de Proyectos Arquitectónicos / ETS Arquitectura / Universidad Politécnica de Madrid / España

Oliverira do Nascimento, Francisco. Professor Auxiliar / Faculdade de Arquitetura / Universidade de Lisboa / Portugal.

Rovira Llobera, Teresa. Titular de Universidad / Departamento de Projectes Arquitectònics / ETS Arquitectura / Universitat Politècnica de Catalunya, Barcelona Tech / España.

Ruiz Rosa, José Antonio. Catedrático de Universidad / Departamento de Expresión Gráfica Arquitectónica / ETS Arquitectura / Universidad de Sevilla. / España.

Sabaté Bel, Joaquín. Catedrático de Universidad / Departamento de Urbanismo y Ordenación del Territorio / ETS Arquitectura / Universitat Politècnica de Catalunya, Barcelona Tech / España

Sainz Gutiérrez Victoriano. Titular de Universidad / Departamento de Urbanismo y Ordenación del Territorio / ETS Arquitectura / Universidad de Sevilla / España

Sánchez Lampreave, Ricardo. Profesor Titular / Área de Composición Arquitectónica / Escuela de Ingeniería y Arquitectura / Universidad de Zaragoza / España.

Sambricio R. Echegaray, Carlos. Catedrático de Universidad / Departamento de Composición Arquitectónica / ETS Arquitectura / Universidad Politécnica de Madrid / España. / España.

Santamarina-Macho, Carlos. España. Doctor Arquitecto, Profesor Asociado / Departamento de Teoría de la Arquitectura y Proyectos Arquitectónicos / ETS Arquitectura / Universidad de Valladolid / España

Senra Fernández-Miranda, Ignacio. Doctor Arquitecto, Profesor Asociado / Departamento de Proyectos Arquitectónicos / ETS Arquitectura / Universidad Politécnica de Madrid / España.

Sentieri Omarrementeria, Carla. Titular de Universidad / Departamento de Proyectos Arquitectónicos / ETS Arquitectura / Universidad Politécnica de Valencia / España.

Sequeira Marta. Professora doctora / ISCTE - Instituto Universitário de Lisboa/ Universidade Autónoma de Lisboa / CIAUD - Faculdade de Arquitectura da Universidade de Lisboa / Portugal.

Sola Alonso, José Ramón. España. Profesor Contratado Doctor / Departamento de Teoría de la Arquitectura y Proyectos Arquitectónicos / ETS Arquitectura / Universidad de Valladolid / España.

Trillo Martínez, Valentín. Doctor arquitecto Profesor Asociado / Departamento de Proyectos Arquitectónicos / ETS Arquitectura / Universidad de Sevilla / España.

Villalobos Alonso, Daniel. Titular de Universidad / Departamento de Teoría de la Arquitectura y Proyectos Arquitectónicos / ETS Arquitectura / Universidad de Valladolid / España.

Verde Zein, Ruth. Doctora arquitecto e investigadora / Facultad de Arquitectura y Urbanismo / Universidad Presbiteriana Mackenzie. São Paulo / Brasil.

\section{ESTADÍSTICAS PUBLICACIÓN (publicación cada cuatro números, dos años). NÚMEROS 1 a 20 (incluidos)}

Total artículos recibidos: 546

Total artículos publicados: 199 (36,45\%)

Total artículos rechazados: $347(63,55 \%)$

Total artículos publicados de autores pertenecientes a los diferentes consejos o comités organizadores de la revista y Grupo de Investigación "proyecto, progreso, arquitectura"(endogamia): 20 (10,05\%)

Total artículos publicados de autores pertenecientes a la Universidad de Sevilla: 53 (26,63\%)

Total artículos publicados de autores externos a los diferentes consejos o comités organizadores de la revista y Grupo de Investigación

"proyecto, progreso, arquitectura": 179 (89,95\%)

Total artículos publicados de autores extranjeros: 17 (8,54\%) 


\section{arquitecturas ampliadas}

índice

editorial

DESPEJAR LA ARQUITECTURA, LIBERAR EL ESPACIO Y AMPLIAR CONCEPTOS / UNCLUTTER ARCHITECTURE, FREE UP SPACE AND EXPAND CONCEPTS

Rosa María Añón-Abajas - (DOI: http://dx.doi.org/10.12795/ppa.2021.i24.10)

entre líneas

PAISAJES ARQUITECTÓNICOS Y MEMORIAS DE LA CIUDAD / ARCHITECTURAL LANDSCAPES AND MEMORIES OF THE CITY

Darío Álvarez Álvarez - (D0l: http://dx.doi.org/10.12795/ppa.2021.i24.01)

artículos

LINAZASORO EN REIMS. EL ESPACIO PÚBLICO COMO MEMORIA DEL LUGAR / LINAZASORO IN REIMS. THE PUBLIC SPACE AS THE MEMORY OF THE PLACE

Victoriano Sainz Gutiérrez - (DOl: http://dx.doi.org/10.12795/ppa.2021.i24.02)

EDITAR VS. CONSTRUIR: UNA ECOLOGÍA DE LO INVISIBLE. AMPLIFICAR LA COMPRENSIÓN DE LAS TÉCNICAS DE PROYECTO / EDITING VS. BUILDING: AN ECOLOGY OF THE INVISIBLE. AMPLIFYING THE UNDERSTANDING OF ARCHITECTURAL DESIGN TECHNIQUES

Paula Victoria Álvarez Benítez - (DOI: http://dx.doi.org/10.12795/ppa.2021.i24.03)

CUANDO LA PINTURA AMPLÍA LA ARQUITECTURA: INTERVENCIONES REALIZADAS EN EL ESPACIO PÚBLICO / WHEN PAINTING ENHANCES ARCHITECTURE: INTERVENTIONS IN A PUBLIC SETTING Aurora Alcaide-Ramírez; Ana Ruiz-Abellón - (D0l: http://dx.doi.org/10.12795/ppa.2021.i24.04)

UN EDIFICIO INVISIBLE. NUEVO AULARIO DE LA FACULTAD DE DERECHO DE LA UNIVERSIDAD DE ZARAGOZA (1983-1996) / AN INVISIBLE BUILDING. NEW LECTURE ROOM BUILDING OF THE FACULTY OF LAW OF THE UNIVERSITY OF ZARAGOZA (1983-1996)

Luis Miguel Lus-Arana; Lucía Carmen Pérez-Moreno - (D0l: http://dx.doi.org/10.12795/ppa.2021.i24.05)

ARQUITECTURAS AMPLIADAS. EL PABELLÓN DE EXPOSICIONES EN LA CASA DE CAMPO DE MADRID) / EXPANDED ARCHITECTURES. THE EXHIBITION PAVILION AT THE CASA DE CAMPO IN MADRID José de Coca Leicher - (DOI: http://dx.doi.org/10.12795/ppa.2021.i24.06)

reseña bibliográfica TEXTOS VIVOS

RAFAEL MONEO VALLÉS: LA VIDA DE LOS EDIFICIOS. LA MEZQUITA DE CÓRDOBA, LA LONJA DE SEVILLA Y UN CARMEN EN GRANADA

Víctor Pérez Escolano - (DOl: http://dx.doi.org/10.12795/ppa.2021.i24.07)

FRANCISCO DE GRACIA: CONSTRUIR EN LO CONSTRUIDO. LA ARQUITECTURA COMO MODIFICACIÓN

Pablo Diañez Rubio - (DOl: http://dx.doi.org/10.12795/ppa.2020.i24.08)

FRÉDÉRIC DRUOT, ANNE LACATON \& JEAN-PHILIPPE VASSAL PLUS: LA VIVIENDA COLECTIVA. TERRITORIO DE EXCEPCIÓN

Javier Terrados Cepeda - (DOI: http://dx.doi.org/10.12795/ppa.2021.i24.09) 


\title{
ARQUITECTURAS AMPLIADAS. EL PABELLÓN DE EXPOSICIONES EN LA CASA DE CAMPO DE MADRID
}

\author{
EXPANDED ARCHITECTURES. THE EXHIBITION PAVILION AT THE CASA DE CAMPO IN \\ MADRID
}

José de Coca Leicher (https://orcid.org/0000-0002-0950-4163)

RESUMEN La reciente intervención en el pabellón de Exposiciones, antiguo pabellón del Ministerio de la Vivienda realizado en 1959 para la IV Feria Internacional del Campo por Francisco de Asís Cabrero y Jaime Ruiz, es un interesante ejemplo de estrategias para recuperar arquitecturas, en este caso del siglo XX, ampliando su uso y su vida útil mediante la adaptación de sus espacios interiores y exteriores a nuevas necesidades ciudadanas, exigencias de accesibilidad y de acondicionamiento climático. La rehabilitación, dentro de los "usos compatibles" previstos en el Plan Especial vigente, es parte de la iniciativa Campus Asociativo de Madrid formada por cuatro pabellones: Exposiciones, Valencia, Icona I e Icona II. En ese contexto, analizaremos las estrategias de intervención y las operaciones realizadas, a partir del estudio del edificio original y relacionándolo con otras arquitecturas de referencia, entre otros, los edificios que Mies van der Rohe realizó en el campus del IIT, también restaurados y que inspiraron a Cabrero y Ruiz. Alimentamos el debate de la recuperación de edificios, en el que lo realizado enriquece los criterios al uso. Concluiremos evidenciando el equilibrio entre la recuperación de la espacialidad, su construcción experimental y la transformación del uso expositivo en el nuevo uso representativo y administrativo de carácter permanente, pero reversible, en lo que consideramos una "arquitectura ampliada".

PALABRAS CLAVE arquitecturas ampliadas; pabellón de Exposiciones; Campus Asociativo; Cabrero; Casa de Campo; Madrid

SUMMARY The recent rehabilitation of the Exhibition Pavilion, the former Ministry of Housing Pavilion, carried out in 1959 by Francisco de Asís Cabrero and Jaime Ruiz for the 4th International Country Fair, is an interesting example of a strategy implemented to recover architectures from the past, specifically those of the $20^{\text {th }}$-Century. In this way, the utility and usefulness of both the indoor and outdoor spaces of the buildings are adapted to new urban needs, accessibility requirements, and climate conditioning. The restoration has been implemented following the "compatible uses" envisaged in the current Special Plan as part of the action group's initiative "Campus Asociativo de Madrid," which includes not only this Exhibition Pavilion, but the Icona I and Icona II Pavillions in Valencia as well. In this context, we will analyze the intervention and operation strategies, starting from the assessment of the original building and its relationship with other referential architectures. Some examples of these are Mies van der Rohe's restored works on IIT campus, which inspired Cabrero and Ruiz. We have promoted the debate on the recovery of buildings where the precedent projects improve the guidelines for the building's use. We will conclude by showing the balance between the recovery of space, its experimental construction, and the transformation of the exhibition use into the new representative and administrative function of a permanent, but reversible nature, in what we have coined an "expanded architecture."

KEYWORDS expanded architecture; Exhibition Pavilion; Campus Asociativo; Cabrero; Casa de Campo; Madrid. 
"En efecto. Yo creo que se pueden superponer la curva de la población, del desarrollo antropológico, y una curva continuación del desarrollo de la arquitectura, o más bien de la cultura plena, porque la arquitectura es una máquina fotográfica de la cultura: tiene mucho de ciencia, pero tiene mucho de sentido de vivir..."1.

La rehabilitación del pabellón de Exposiciones, un ejemplo singular de arquitectura moderna española, ejemplifica los retos y dificultades en la praxis de la recuperación y adecuación de edificaciones recientes. En este caso, el espacio expositivo original, junto al interés de la solución constructiva, han de adaptarse a unas nuevas necesidades que obligan a la actualización y la ampliación de lo construido. La solución analizada responde a estas estrategias mediante la introducción de pequeñas arquitecturas, las instalaciones completas para cumplir con condiciones climáticas y de accesibilidad junto a la incorporación de los espacios exteriores. La operación Campus Asociativo de Madrid afecta a otros tres edificios con distinto valor y grado de protección y, a su vez, se implanta en el recinto Ferial de la Casa de Campo de Madrid, un ámbito protegido de escala ciudad, con una historia y unos condicionantes que obligan a un recorrido previo (figura 1).

\section{ORIGEN, CONTEXTO Y SITUACIÓN}

\section{ACTUAL DEL RECINTO}

El recinto Ferial de la Casa de Campo de Madrid tiene su origen en las Ferias del Campo que se celebraron entre 1950 y 1975 para potenciar y modernizar la agricultura y la ganadería. Los arquitectos Francisco de Asís Cabrero y Jaime Ruiz proyectaron un primer recinto de $15 \mathrm{ha}$, donde se inauguró la primera Feria Nacional del Campo en 1950 que se resolvió con estands de bóvedas y arcos de ladrillo, una plaza circular de acogida y una moderna torre-mirador orientada hacia la cornisa de Madrid. Hoy solo se conservan unas pocas edificaciones y el pinar hacia el río Manzanares².

En 1953 se inauguró la primera Feria Internacional del Campo. Cabrero y Ruiz diseñaron también el segundo recinto, de 55 ha, que ampliaba el primero hacia el oeste. 


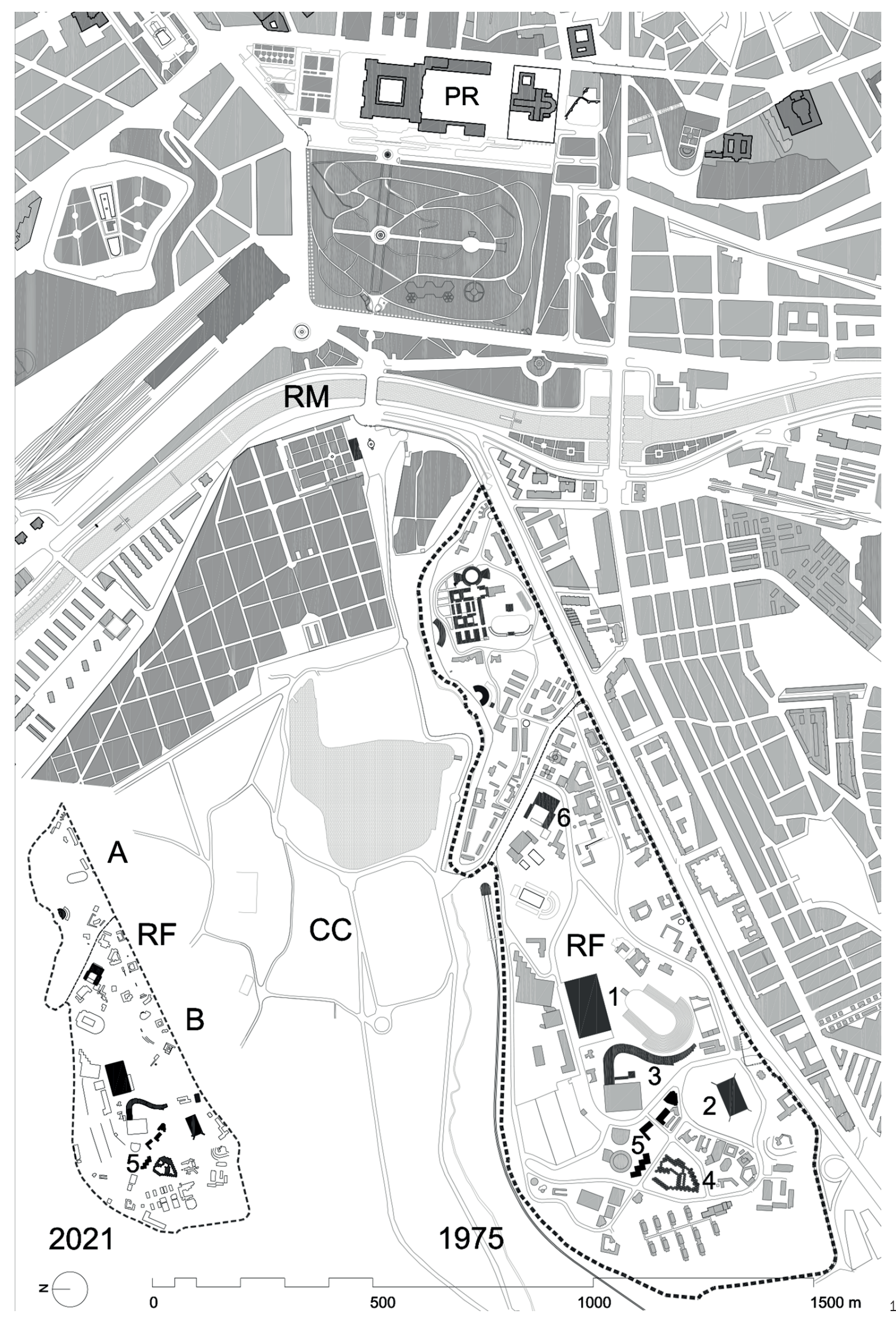


1. El recinto, pabellones representativos y relación con la cornisa histórica y el río Manzanares. PR Palacio Real. RM Río Manzanares. CC Casa de Campo. RF Recinto Ferial. A Recinto de la IFNC. B Recinto de la FIC. 1 Pabellón de Cristal. 2 Pabellón Internacional. 3 Palacio de la Agricultura. 4 Pabellón de Hexágonos. 5 Pabellón de Exposiciones (Ministerio de la Vivienda). 6 Escuela de Hostelería. 2. Campus Asociativo de Madrid.. 1 Pabellón Icona II, 2 Pabellón Icona I, 3 Pabellón de Valencia, 4 Pabellón de Exposiciones, 5 Pabellón de Hexágonos, 6 Teatro Auditorio.

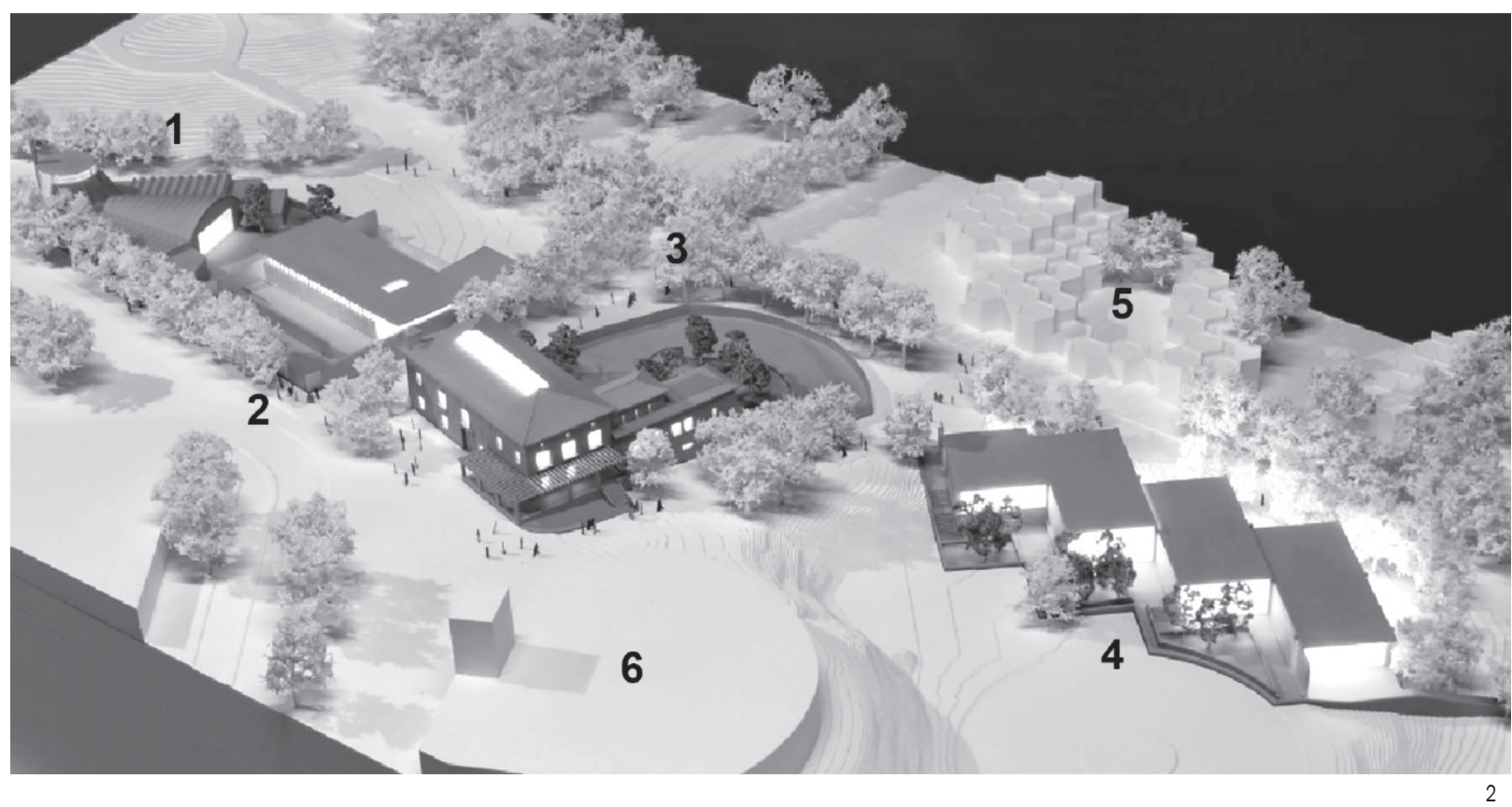

Un bello trazado paisajístico con avenidas arboladas y parcelas donde se situaron los pabellones regionales. Destacaron los pabellones realizados por los pioneros: Miguel Fisac, el de Ciudad Real; José Luis Romaní, el de Jaén y Alejandro de la Sota, el de Pontevedra, arquitecturas modernas desaparecidas inspiradas en lo popular.

Otros pabellones representativos realizados por $\mathrm{Ca}-$ brero y Ruiz, conservados y protegidos, estructuran este recinto; como el del Ministerio de la Vivienda -objeto de nuestra investigación-, el de la Obra Sindical del Hogar, el Palacio de la Agricultura y el pabellón Internacional (figuras 1 y 3). Con la finalización de las Ferias, en 1977 se censan 123 edificios ${ }^{3}$, creándose en 1978 la Dirección de Instalaciones Feriales. En 1980 se funda el Patronato Municipal para el aprovechamiento de las instalaciones del recinto de la Feria del Campo ${ }^{4}$.
Un primer catálogo e inventario fotográfico fue la información urbanística ${ }^{5}$ previa al primer Plan General de Ordenación democrático en 1985. Buscando la continuidad con la Casa de Campo se realizaron "labores de esponjamiento", demoliéndose las bóvedas de la primera Feria. Debido a la inoperatividad del Patronato y al abandono del recinto, se funda en 2001 la Empresa Municipal Campo de las Naciones, iniciándose el Plan Especial "Feria del Campo", aprobado definitivamente en 2006.

Para revitalizar el recinto y tratando de ejecutar el Plan Especial, en 2006 se crea Madrid Espacios y Congresos. Se impulsó la zona de gastronomía en los pabellones regionales y la actividad expositiva en los pabellones representativos, muy disminuida con el traslado de la Feria de Madrid (IFEMA) al parque Juan Carlos I. Después de los

3. Ibídem, p. 22. Realizado por el arquitecto Luis Labiano. Las 123 edificaciones se valoraron en 2059800000 pesetas.

4. Estudios Previos Plan Especial "Feria del Campo" AOE 00.04. Vol. 1 Memoria. Ayuntamiento de Madrid, 2002.

5. Archivo IFEMA. Información Urbanística Básica Recinto Ferial de la Casa de Campo Madrid. Ayuntamiento de Madrid, diciembre 1984. Javier de la Calzada y Juan Carlos García Barbería, arquitectos. 


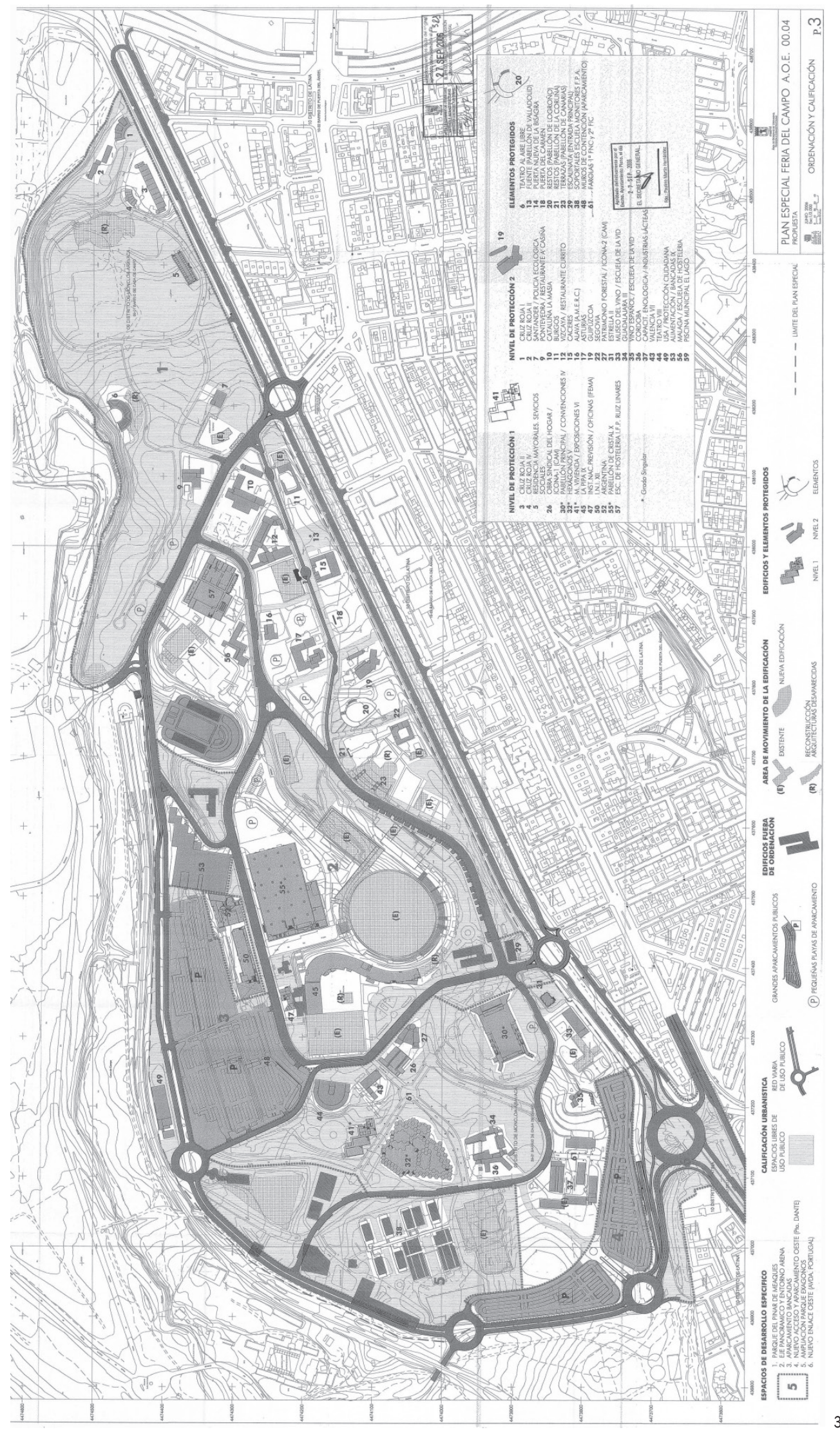


sucesos del pabellón Madrid Arena ${ }^{6}$, en 2013, el recinto Ferial es absorbido por la empresa Madrid Destino Cultura Turismo y Negocio, que es su actual gestora.

Después de 45 años, el trazado original se conserva alterado sobreviviendo el $40 \%$ de los pabellones originales. Salvo el conocido pabellón de Cristal y el antiguo pabellón Internacional (actual de Convenciones), ambos con Nivel 1 Grado Singular, restaurados y que mantienen el uso expositivo, muchas edificaciones emblemáticas situadas en el núcleo del recinto, como el pabellón de la Pipa, antiguo Palacio de la Agricultura, están ahora ocupadas por los servicios del 112 y la Policía municipal en virtud del Plan conocido como la "Ciudad de las Emergencias"7. Ante la falta de una idea motriz que implemente un uso público viable, muchos pabellones alojan usos inapropiados o están vacíos.

\section{ESTRATEGIAS DE RECUPERACIÓN: EL PLAN ESPECIAL "FERIA DEL CAMPO" Y EL CAMPUS ASOCIATIVO DE MADRID}

El Plan Especial ${ }^{8}$ apostaba por la especialización del ámbito con una identidad propia, de uso intensivo del parque histórico para exposiciones complementarias a IFEMA, compatible con usos culturales, dotacionales y de ocio ${ }^{9}$. Se centraba en la rehabilitación de pabellones, potenciando los espacios e itinerarios peatonales, disminuyendo el tráfico rodado e incorporando las actuaciones de Madrid Río y el parque histórico, tratando, además, de vincular los barrios limítrofes.
El catálogo de edificios y elementos arquitectónicos fijó el estado de conservación, el nivel y grado de protección de 38 pabellones. Cada ficha aportaba la planta, una descripción del pabellón original con los valores destacables, los elementos de mayor calidad, los añadidos que eliminar y los elementos que restaurar ${ }^{10}$. Se catalogaron con Nivel 1 Grado Singular cuatro pabellones por su alto valor arquitectónico y referencial. Con Nivel 1 Grado Integral se protegieron nueve pabellones. Con Nivel 2 Grado Estructural otros 25 pabellones. Finalmente, se protegieron once elementos de pabellones desaparecidos y las farolas "constructivistas", diseñadas por Cabrero para la segunda Feria"11 (figura 3).

En la anterior legislatura municipal ${ }^{12}$ se promovió el proyecto Campus Asociativo de Madrid $^{13}$ con un doble objetivo: recuperar los edificios del recinto para el patrimonio cultural del siglo XX y dotar a la ciudad de una infraestructura general. Los cuatro pabellones escogidos, incluido el de Exposiciones, llevaban años sin uso, con la consiguiente pérdida funcional y patrimonial.

El pabellón Icona II, cubierto con unas atractivas ondas de hormigón, representó a la D. G. de Montes y fue realizado en 1957 por Eduardo Baselga, es Nivel 2 Grado Estructural. El pabellón de Icona I era sede de la Obra Sindical del Hogar y fue realizado por Francisco Cabrero y Felipe Pérez Enciso. Inspirado en el pabellón de Barcelona de Mies van der Rohe, dispone de un mural cerámico restaurado de los artistas Amadeo Gabino y Manuel Suárez Molezún, es Nivel 1 Grado Integral. El pabellón

6. El trágico accidente que acabó con la vida de cinco adolescentes el 1 de noviembre de 2012.

7. Plan Especial de pormenorización de usos y obras en diversos edificios protegidos de la zona ferial de la Casa de Campo. Ayuntamiento de Madrid: Área de Gobierno de Economía, Hacienda y Administración Pública, 2014 [consulta 29-09-2020]. Disponible en: ftp://rfa.munimadrid.es/PContratante/publicados/0 140e2dba4645410VgnVCM1000000b205a0aRCRD/1397117287839/CIUDAD_SEGURIDAD_02.pdf.

8. El P. E. “Feria del Campo" AOE.00.04 se adjudica en 2004 y se aprueba definitivamente en 2006. Dirigido por J. Gago y J. M. García-Pablos, el catálogo fue realizado por José de Coca, arquitectos. El Plan desarrolla lo fijado en el PGOUM de 1997. Mención en los premios de Urbanismo, Arquitectura y Obra Pública 2006 del Ayuntamiento de Madrid.

9. Plan Especial "Feria del Campo" AOE.00.04. Madrid: Ayuntamiento de Madrid, 2006.

10. Ibíd. Anexo 1: Catálogo de Edificios y Elementos.

11. COCA, José de, op. cit. supra, nota 2, p. 268. Lamentablemente, aun estando protegidas, han sido eliminadas y sustituidas por las tradicionales farolas tipo "fernandina".

12. Entre junio de 2015 y junio de 2019, siendo alcaldesa Manuela Carmena.

13. Dosier Campus Asociativo de Madrid. Madrid: Coordinación territorial y cooperación público social, 2018 [consulta 29-09-2020]. Disponible en: https:// diario.madrid.es/wp-content/uploads/2018/12/Dosier-Campus-Asociativo-de-Madrid.pdf 


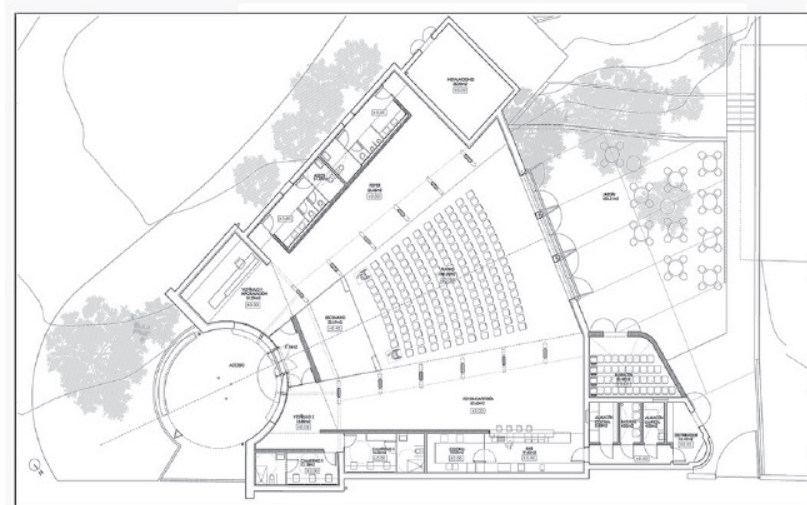

1

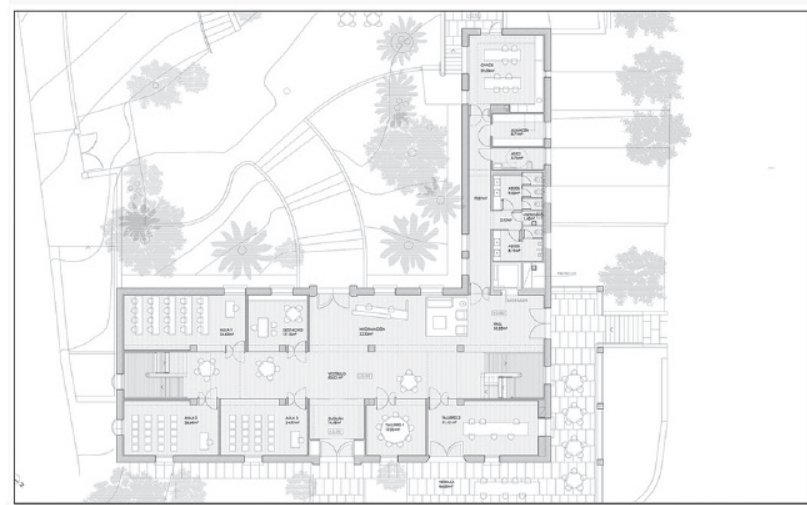

2
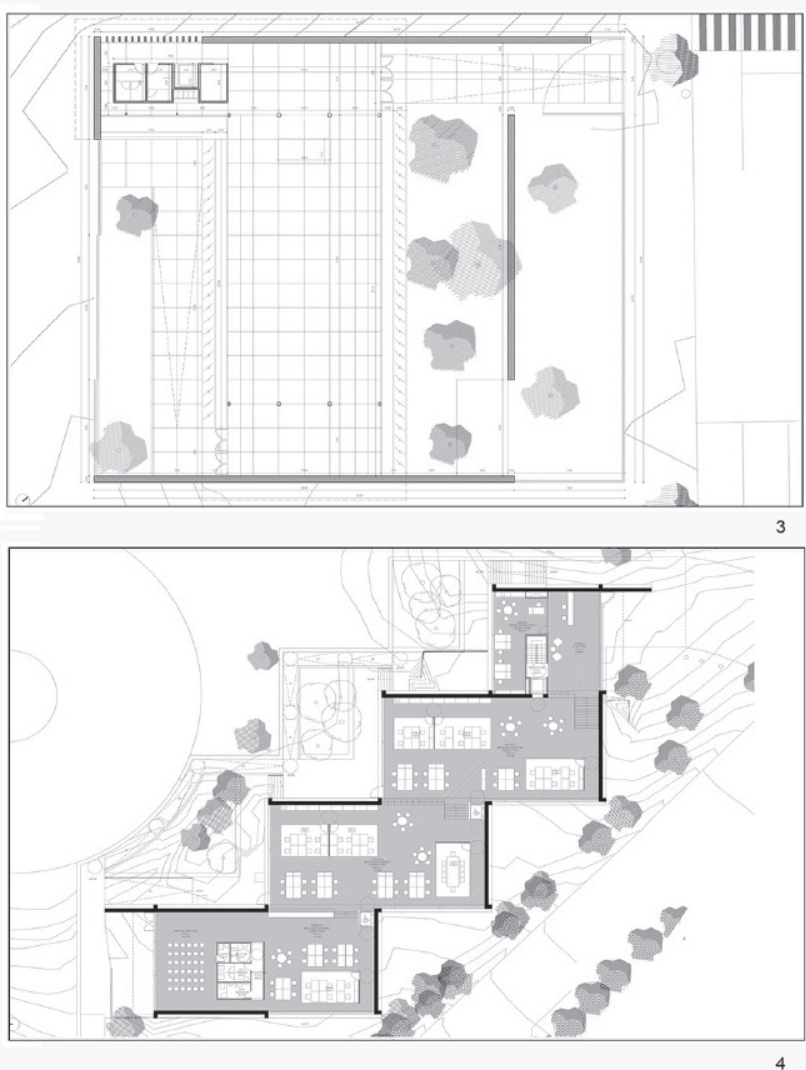

de Valencia VII es regionalista y su protección es Nivel 2 Grado Estructural, ambos son de 1959.

El pabellón de Exposiciones, edificio principal del Campus, fue el pabellón del Ministerio de la Vivienda, proyectado en 1959 por Francisco Cabrero y Jaime Ruiz. Su nivel de protección es el máximo, es decir, Nivel 1 Grado Integral. Es un edificio de gran valor arquitectónico y es un hito imprescindible en la evolución de la trayectoria de Francisco Cabrero y de la arquitectura moderna madrileña del siglo XX (figuras 2 y 3 ).

El programa del Campus se distribuyó según las arquitecturas, la idoneidad funcional y el régimen de obras permitido ${ }^{14}$. El pabellón Icona I, por su sala diáfana: exposiciones y biblioteca. El pabellón de Valencia, de menor valor, pero mayor funcionalidad: talleres, coworking, comunicación y reunión. El pabellón Icona ll: equipamiento cultural con espacio escénico y cafetería. Finalmente, el pabellón de Exposiciones, el más representativo: las sedes de las federaciones, zonas de trabajo y asesoramiento, con el reto de mantener su extraordinaria espacialidad con un uso compartimentado (figura 4).

\section{EL PABELLÓN DEL MINISTERIO DE LA VIVIENDA. LOS PRINCIPIOS DE LA "EDAD DEL HIERRO"}

El pabellón del Ministerio, realizado en colaboración con Jaime Ruiz, es la primera de una serie de obras de Cabrero, realizadas desde finales de los 50 hasta mediados de los 60, en las que reduce su lenguaje expresivo a unos pocos materiales. Cabrero reconoce la influencia de los edificios de Mies van der Rohe del campus del Illinois Institute of Technology (ITT), visitados en el año 1956.

En una entrevista donde también valora el origen neoplasticista de su obra ${ }^{15}$, define esta como una combinación entre la descomposición de planos, la forma cúbica y el orden ortogonal para responder a las "tres imaginaciones" de la arquitectura: espacio, materialidad y trazado ${ }^{16}$.

14. Dosier Campus Asociativo, op. cit. supra, nota 13, pp. 13-14.

15. MATA, Sara de la; SOBEJANO, Enrique. Entrevista Francisco de Asís Cabrero. En: Arquitectura. Madrid: COAM, 1987, n. ${ }^{2}$ 267, pp. 110-115.

16. Ver CABRERO, Francisco de Asís. Las tres naturalezas del neoplasticismo. En: Los cuatro libros de la Arquitectura. Libro III. Madrid: COAM, 1992, pp. 342343. 
4. Campus Asociativo de Madrid, 2018. Proyectos de restauración y nuevos usos: 1 Pabellón Icona II, 2 Pabellón de Valencia, 3 Pabellón Icona I (Obra Sindical del Hogar), 4 Pabellón de Exposiciones (Ministerio de la Vivienda).

5. Pabellón del Ministerio de la Vivienda. Francisco de Asís Cabrero y Jaime Ruiz, 1959.
Este pensamiento, basado en el carácter abstracto del neoplasticismo, le acerca al trabajo del artista y arquitecto Max Bill, una figura admirada que alcanza la integración de todas las artes, plasmada en sus obras con un concepto unificador o "arte concreto" en el que Cabrero basa su noción de constructivismo (figura 5).

En esta etapa, que define irónicamente como la "edad del hierro", Cabrero reduce su lenguaje a las propiedades del acero y a la combinación de los perfiles industriales. Somete la arquitectura al lenguaje de las retículas de acero, estudiando las uniones y confiando en la esbeltez del material que le permite realizar enormes dinteles ${ }^{17}$. El espacio, por tanto, se genera por la combinación de entramados que salvan grandes vanos y los cerramientos con materiales idóneos en cuanto a su función y estética: el ladrillo y el vidrio.

Para Cabrero, la característica "trascendental" de la arquitectura del siglo XX es su significado "utilitario y social". El edificio debe facilitar la "vida programada", lo que supone una perfección del orden social por medio de unas formas plásticas "experimentadas y de continuidad"18. Define "útil" como algo provechoso, conveniente y ventajoso que resume en el vocablo "funcional", asociando al contenido la solución del programa, y al continente, el funcionamiento apropiado de la mecánica constructiva y la estructura.

Cada programa estará asociado a una determinada solución constructiva y estructural "a su propia mecánica y aparejo", que resolverá el interior y exterior del edificio mediante su expresión plástica. Siguiendo la definición de "arte concreto" de Max Bill, Cabrero denomina "constructivismo" al término que relaciona el aparejo, la lógica estructural, la modulación y los materiales. Está basado en las decisiones lógicas de proyecto, rastreables en el edificio terminado y alcanzadas expresando la idea

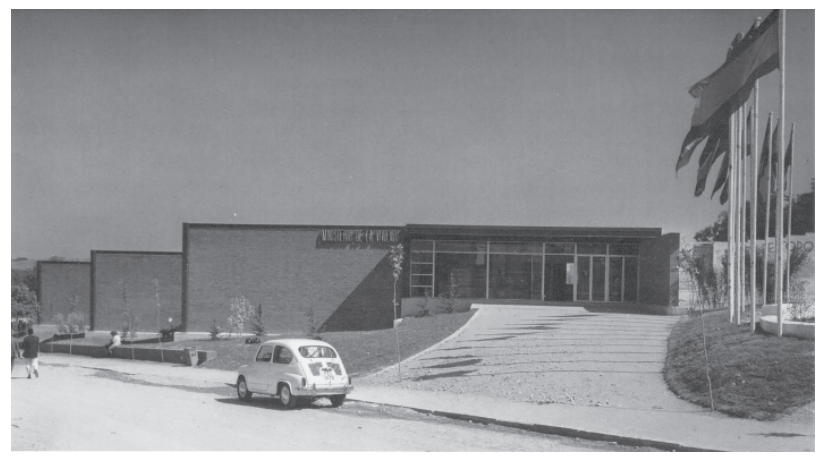

5

principal al activar las cualidades plásticas del espacio y la materia. El ideal clásico permanece como una ley de proporción y relación entre partes, incorporando la perspectiva central o el escorzo desde una visión moderna y dinámica, basada en las "tres naturalezas del neoplasticismo" aludidas.

La arquitectura es un arte, pero con el fin de alcanzar la "belleza útil". Un sentido utilitario condicionado por "unas necesidades directas que el hombre tiene y que precisan ser satisfechas, pero no de cualquier modo" y mientras tanto "la arquitectura tiene que hacer palpable que es un arte"19. En la construcción con acero es donde se consigue expresar la condición "constructivista" y experimental de la arquitectura ${ }^{20}$. Una concepción directa y desnuda que Cabrero define como la "arquitectura desornamentada"21 (figuras 6 y 7 ).

El conocimiento del material es imprescindible para el arquitecto: "No se conoce bien la madera [...] hasta que no se trabaja. Lo mismo ocurre para el hierro, la piedra y los demás materiales"22. Cabrero, en este periodo, pinta las estructuras de hierro en rojo, el rojo presente en la naturaleza: "Probé tonos minio, luego diferentes rojos en

17. "La retícula y el hierro", ver: RUIZ CABRERO, Gabriel. Vida y obra de Asís Cabrero. En: AAVV, Francisco de Asís Cabrero. Legado 02. Madrid: Fundación COAM, 2007, p. 67.

18. CABRERO, Francisco de Asís. Francisco de Asís Cabrero. Título 1942. En: Arquitectura. Madrid: COAM, 1964, n. ${ }^{\circ}$ 61, p. 16.

19. CASTRO, op. cit. supra, nota 1, p. 6.

20. SÁNCHEZ DE LA CHICA, Juan Manuel. Materia, material y aparejo en la arquitectura de Francisco de Asís Cabrero. Director: Gabriel Ruiz Cabrero. Tesis doctoral, ETS de Arquitectura Universidad de Málaga, 2015, p. 342.

21. Ver: "arquitectura desornamentada". CABRER0, op. cit. supra, nota 16, p. 60.

22. CASTRO, op. cit. supra, nota 1, p. 5. 


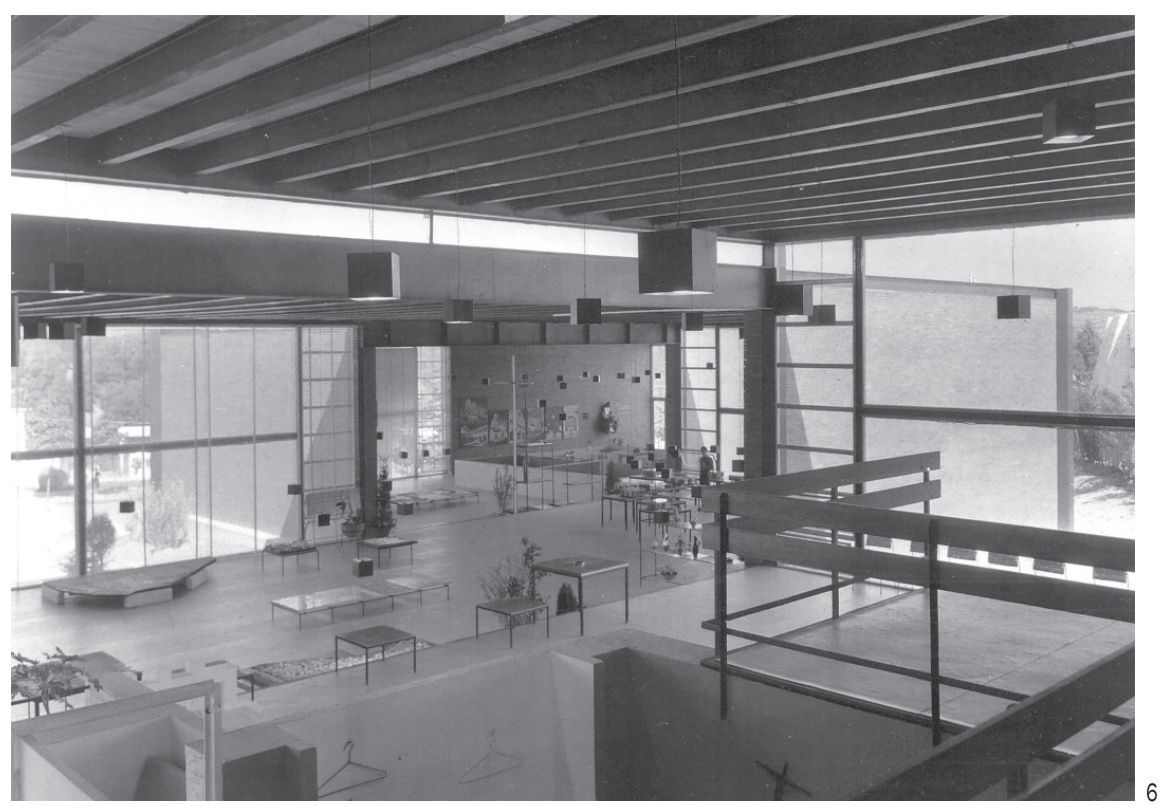

6. Espacio interior diáfano y dinámico del Pabellón del Ministerio de la Vivienda, 1959.

7. Versión construida del pabellón del Ministerio de la Vivienda, 1959.

todas mis obras, y aquí en mi casa me decidí por un rojo más vivo y creo que combina bien con el tono de la madera. Algunas gentes me decían que era un color japonés y a mí no me lo parecía. He comprobado que tenían razón [...] creo que la arquitectura japonesa es eminentemente constructivista"23.

El pabellón del Ministerio de la Vivienda es la primera obra en que Cabrero experimenta los principios expuestos. Previamente, en la Escuela Nacional de Hostelería, realizada con Jaime Ruiz en el recinto ferial en el año 1956 y aún en funcionamiento, utiliza el hierro pintado de negro y exento en la estructura de la fachada ensayando la diferenciación del programa con edificios de distinto material y sistema constructivo.

Posteriormente al pabellón del Ministerio, realiza varias obras donde ensaya variaciones de la misma solución "constructivista". En el edificio para el diario Arriba (1962), el bloque de oficinas paralelo a la Castellana es una retícula metálica de $11 \times 11$ módulos de despachos con entrepaños de ladrillo y vidrio. En la parte trasera, se adosa una estructura fabril de pórticos inclinados y revestimiento de fibrocemento. En su casa, Puerta de Hierro II (1962), fundada sobre un basamento de hormigón, dispone los pilares y vigas metálicas en voladizo sobre el plano de fachada, entrepaños de ladrillo o madera, vidrio, aluminio y cubierta de aluminio.

\section{EL PROYECTO DEL PABELLÓN ORIGINAL}

Se conserva un anteproyecto en el que se plantea un único espacio diáfano de $30 \times 15 \mathrm{~m}$ en planta y $5 \mathrm{~m}$ de altura $^{24}$ y en el que observamos la relación directa con los edificios del Campus del IIT, especialmente la Carr Chapel, recientemente restaurada ${ }^{25}$. Constructivamente, el rectángulo interior se limita en planta con dos cerramientos en forma de "L", uno de ladrillo visto y otro de vidrio con carpintería de aluminio. El entramado de vigas de acero que asoman en cubierta y definen el interior apoya en un extremo en el muro de ladrillo (igual que en la capilla) y el otro en el suelo mediante semipórticos en forma de "L" con la sección en ménsula utilizada en otros pabellones de la Feria (figura 8).

\section{3. Íbid, p. 6.}

24. Pabellón del Ministerio de la Vivienda, IV Feria Internacional del Campo. Casa de Campo Madrid. Legado 02 Francisco de Asís Cabrero. Fundación COAM, 2007. FCT/P104, FCT P449.

25. HARBOE, Gunny. Restoring the "God Box": Mies van der Rohe's Carr Chapel at IIT. En: Ludwig MIES VAN DER ROHE et al. The Heritage of Mies. Eindhoven: Eindhoven Docomomo International Secretariat, 2017, n. ${ }^{0} 56$ (2017/1), pp. 73-77. 

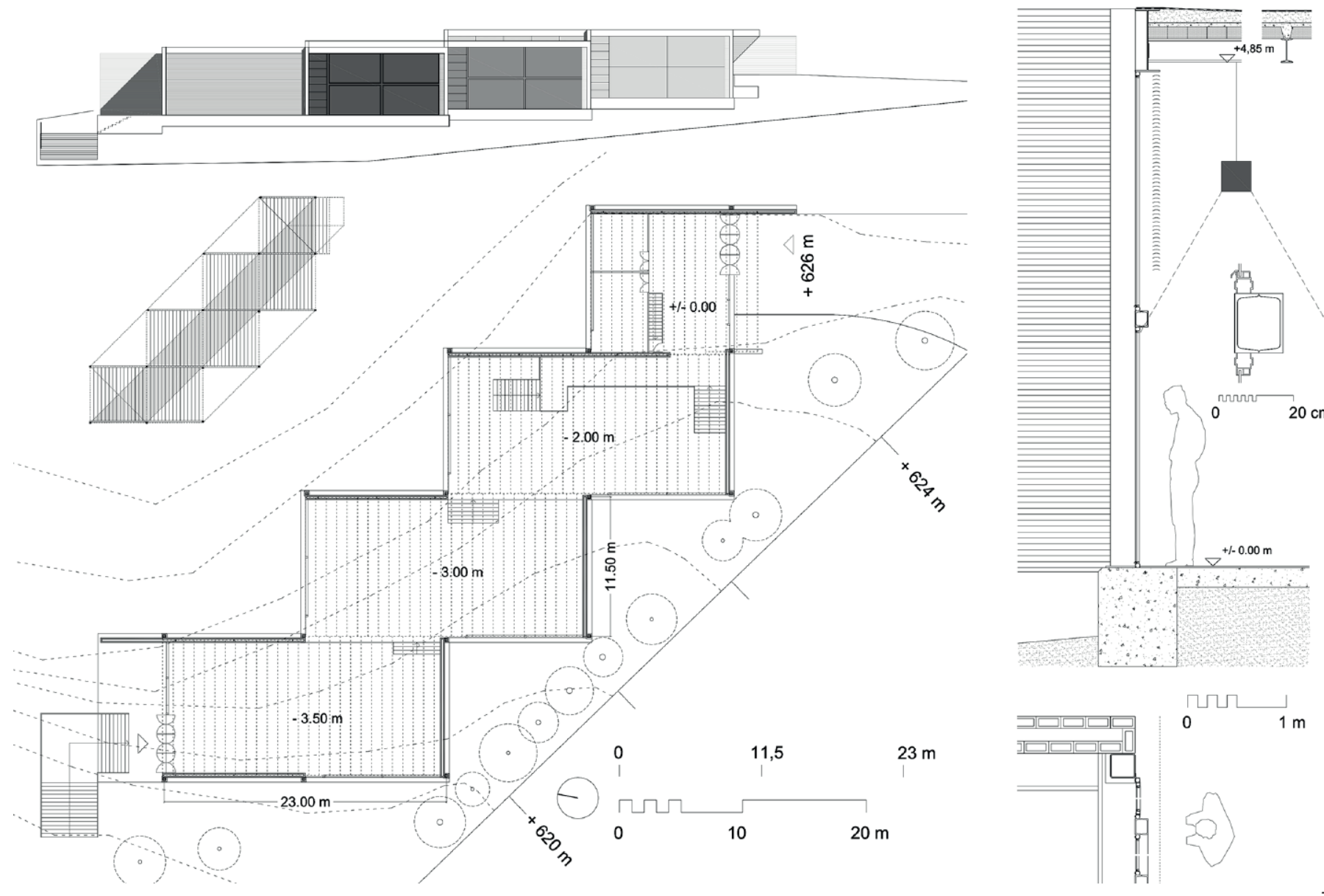

El pabellón definitivo, del que no se conservan los planos del proyecto ${ }^{26}$, se adapta a un nuevo solar con $6 \mathrm{~m}$ de desnivel en perpendicular y a lo largo de la calle. Enfrente se realiza la instalación del conocido pabellón de Bruselas, estableciéndose un interesante diálogo entre sus arquitecturas resueltas con los mismos materiales, pero basadas en sistemas geométricos muy diferentes: el ortogonal frente al hexagonal (ver figura 1).

Se resuelve con tres naves y media. La nave tipo es un rectángulo en planta de $23 \mathrm{~m} \times 11,5 \mathrm{~m}^{27}$, el doble

26. En el estudio de Cabrero solo quedaba el registro de la numeración (planos 2791 a 3840), tampoco se conservó ninguna memoria, solo copias de algunas fotografías. Ver: COCA, José de, op. cit. supra, nota 2, p. 315.

27. Medidos en obra a ejes de pilares. En las dos naves centrales cada tramo en sentido longitudinal son $11,52 \mathrm{~m}$ y la anchura $11,50 \mathrm{~m}$. En la seminave de acceso, la longitud se acorta a 11,36 m y la anchura aumenta a 11,82 $\mathrm{m}$. En la nave inferior, la longitud del último tramo se reduce a 11,28 $\mathrm{m}$ y la anchura a $11,35 \mathrm{~m}$. Estos ajustes del módulo tipo de 11,50 m responden al uso de cada espacio y a la posición relativa entre pilares y vigas debida a la unión tangente 0 "a tope" entre ellos. 

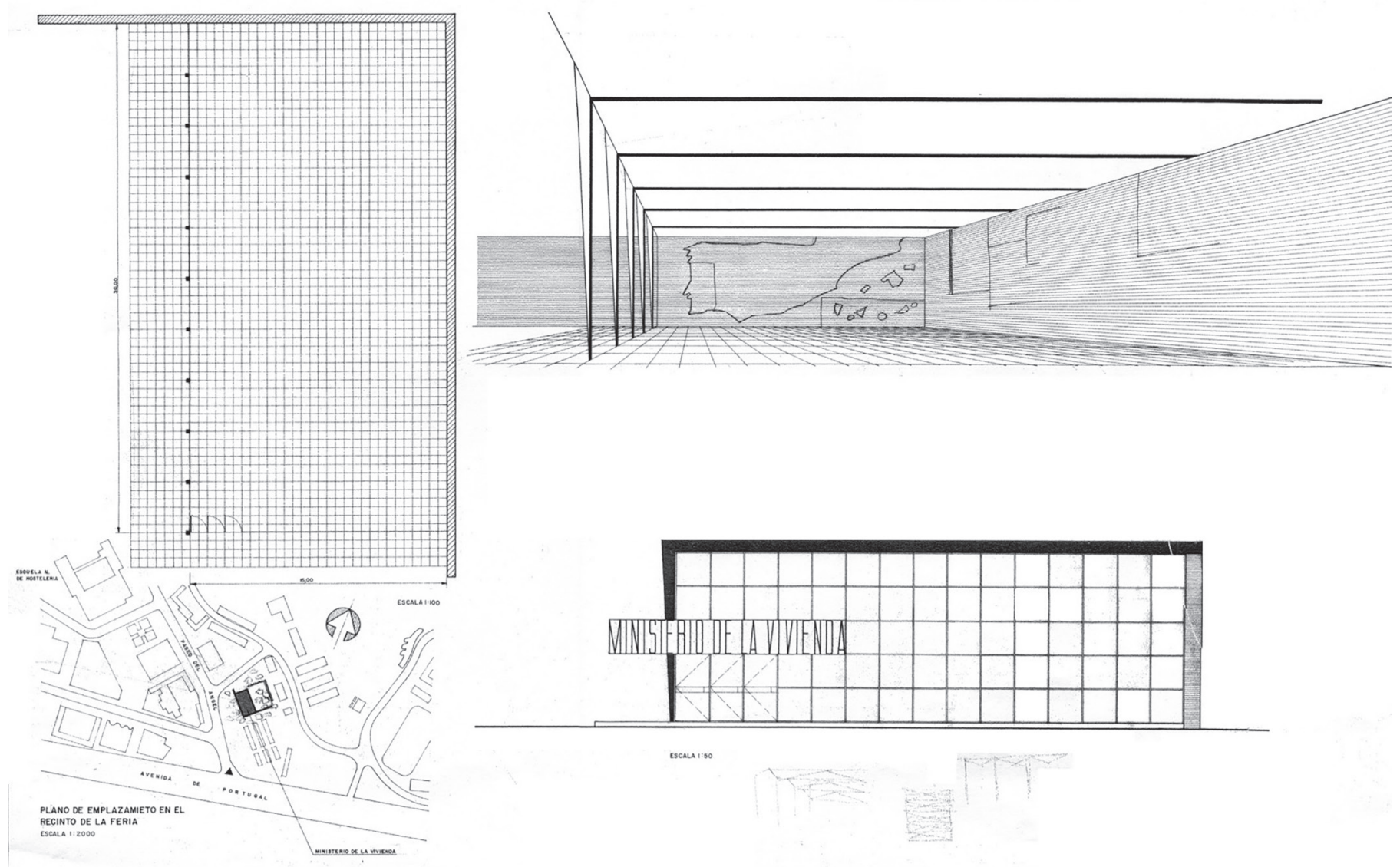

8

cuadrado frecuentemente utilizado por Cabrero. La altura interior vuelve a ser de $5 \mathrm{~m}$, manteniéndose el concepto espacial del anteproyecto. Las naves se escalonan en planta y sección para adaptarse a la alineación de la calle y a la topografía. En planta, el desfase es de 11,50 m, la mitad de su longitud y en sección, al situar la entrada en el punto más elevado, los bancales descienden sucesivamente de mayor a menor altura: 2, 1 y 0,50 m (figura 9).

Estructuralmente, las naves funcionan como pabellones independientes con vigas longitudinales compartidas en cada lado mayor. Consisten una doble "T" acartelada de $635 \mathrm{~mm}$ de canto y $250 \mathrm{~mm}$ de ala, formando pórticos de $11,50 \mathrm{~m}$ de luz y apoyadas en los pilares cuadrados
8. Anteproyecto del pabellón del Ministerio de la Vivienda.

9. Pabellón del Ministerio de la Vivienda, 1959. Elementos definidores de su arquitectura.

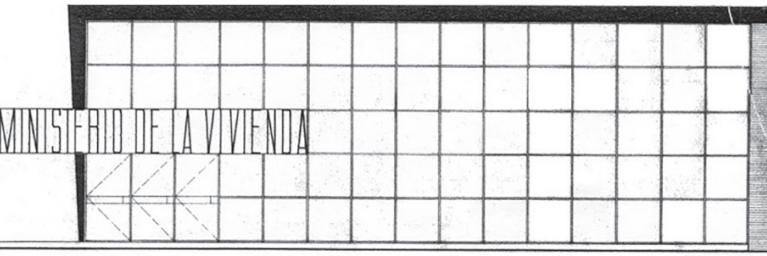

iscana : :so

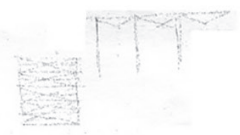

de acero de $260 \mathrm{~mm}$ de lado. Solo el pórtico entre la $3 .^{\mathrm{a}}$ y la $4{ }^{\text {a }}$ nave tiene una viga doble " $T$ " acartelada de 1150 $\mathrm{mm}$ de canto. Perpendicularmente, en los vanos. de $11,50 \mathrm{~m}$, se disponen las viguetas IPN de $240 \mathrm{~mm}$. Como ocurre en otras obras de Cabrero, para poder "acodalar" los muros capuchinos de $1^{1 / 2}$ pie de ladrillo visto y no duplicar vigas, la estructura se resuelve mediante uniones tangentes de vigas y pilares o uniones "a tope".

Existen dos versiones publicadas de la planta que, comparadas con las fotografías, hacen concluir que la planta construida es la de la monografía de $1979^{28}$, en la que se plantea un corredor elevado en el paso de la nave de entrada desde donde se contemplaría el espacio 

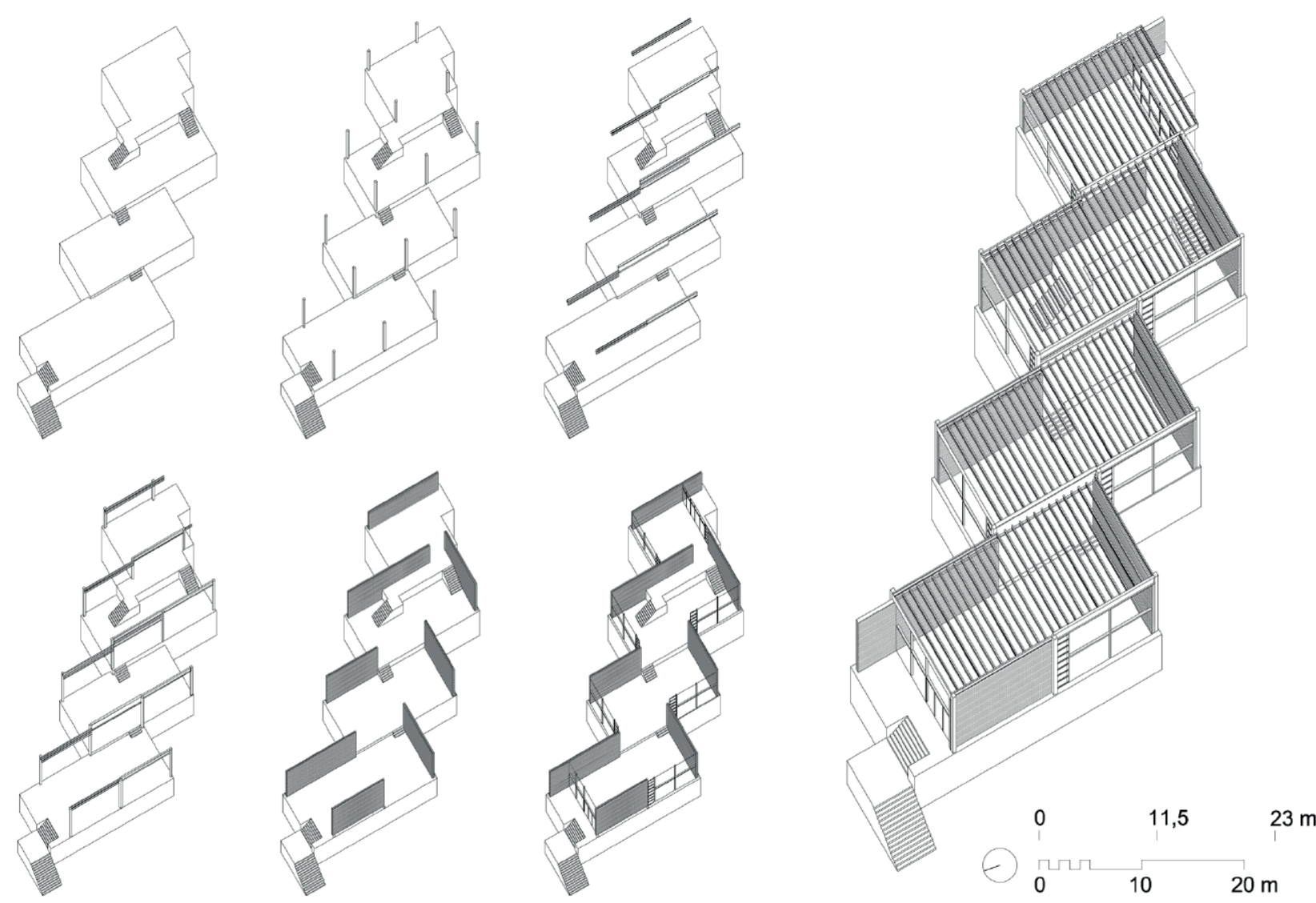

interior completo. Sin embargo, en el proyecto de rehabilitación se tomó como modelo la planta publicada en la revista Arquitectura ${ }^{29}$ en la que se suprime el corredor elevado, se amplía el ancho de la escalera en la 2. ${ }^{a}$ nave y se desplaza la escalera de la tercera nave trasladando el recorrido perimetral a la derecha (figura 10).

El pabellón se cimenta con zapatas de hormigón que forman los bancales y sobresalen del terreno. Encima de estos se apoyan directamente los pilares sobre las placas de reparto y los muros de ladrillo. Las naves comparten las vigas longitudinales a las que acometen las correas con un intereje de $83 \mathrm{~cm}$ y relleno de bloques de hormigón ligero de 12,5 cm de altura, capa de compresión y hormigón de pendiente. La cubierta era una lámina asfáltica con caída hacia unas cazoletas en el centro de cada nave desde donde salía la red horizontal, con las bajantes ocultas en la cámara de los muros de ladrillo.

El cerramiento alternaba los muros de ladrillo visto sin junta con unos enormes paños de vidrio, ambos de
$11,5 \mathrm{~m}$ de ancho $\times 5 \mathrm{~m}$ de alto. Los huecos, excepto una banda lateral de 1,25 $\mathrm{m}$ de ventanas practicables, se dividían en vertical y horizontal mediante una cruz estructural pintada de rojo de 2 UPN de $160 \mathrm{~mm}$, cerrando los entrepaños con carpintería fija de aluminio natural y un vidrio simple según nuestras investigaciones de $6 \mathrm{~mm}$ y 4,50 m de anchura por 2,40 m de altura. Una solución constructiva parecida con vidrios menores se conserva en la entrada lateral del pabellón de Cristal.

El dinamismo del espacio interior era sorprendente, ya que el solapamiento de naves, dejando un hueco de $11,5 \mathrm{~m} \times 5 \mathrm{~m}$ (igual al de los paños de vidrio y ladrillo), provoca una fuga visual que atraviesa las naves formando un nuevo espacio diagonal, descendente o ascendente según la posición del observador (ver figuras 7 y 9). Cuatro años después, frente al pabellón de Cristal, Cabrero y Ruiz repetirán el interior escalonado en el pabellón de Bancadas con un único plano inclinado de cubierta ${ }^{30}$. 
10. Pabellón del Ministerio de la Vivienda. Versiones publicadas en Arquitectura, 1959, n. ${ }^{0} 7 \mathrm{y}$ en el libro Francisco de Asís Cabrero, arquitecto: 1939-1978. La planta derecha es la finalmente construida.

11. Pabellón del Ministerio de la Vivienda. Interior de la 3. ${ }^{\text {a }}$ nave, 1959.
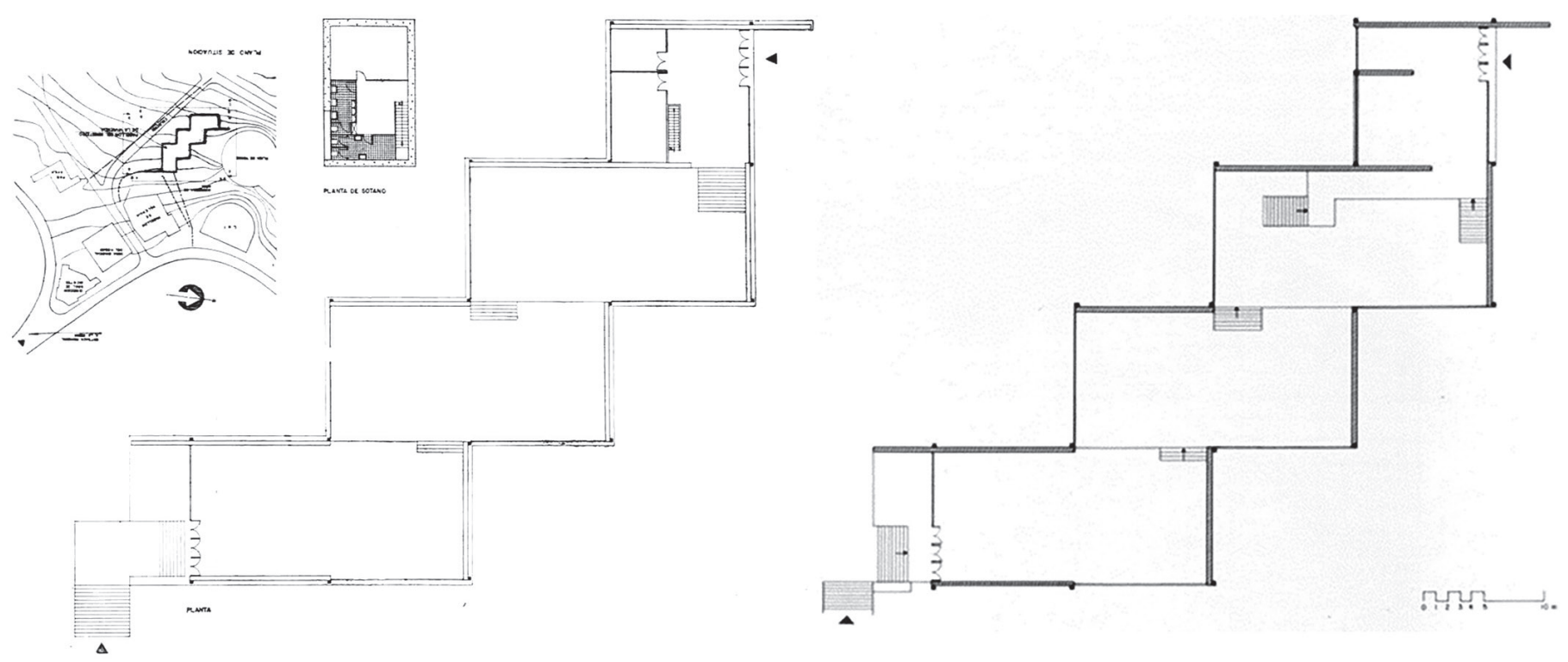

10

Los elementos de mobiliario realizados por la casa Darro eran mesas cuadradas, altas y bajas, con una ligera estructura de tubo rectangular y tablero de madera; igualmente las sillas, que evitaban interferir con la espacialidad interior y repetían la idea del edificio de un elemento apoyado en patas.

Vimos cómo Cabrero asocia el contenido a la solución del programa y el continente a la configuración espacial. Esto provoca cambios de escala entre los paños de ladrillo, las particiones del vidrio y el mobiliario. Cada pared asume su papel de espacio social o espacio íntimo, sirviendo como soporte de información, límite o apertura visual (figura 11).

\section{EL PROYECTO DE REHABILITACIÓN}

En el Plan Especial no permite aumentar la edificabilidad. Los usos característicos de los pabellones están asocia- dos a la actividad ferial, autorizándose temporalmente otros usos, lo que en la práctica supone una ampliación de $u \operatorname{sos}^{31}$ que en el pabellón se concretan en sede de asociaciones y federaciones ${ }^{32}$. En la ficha del catálogo se destacaban como elementos de mayor valor "la diafanidad y comunicación visual entre salas. La racionalidad de la estructura y el cerramiento" y se fijaba "eliminar las galerías al noroeste, tabiquería interior. Restaurar cubiertas, cerramientos de ladrillo. Añadir cerramientos de vidrio respetando la solución original. Recuperar acceso original"33 (figura 12).

En proyecto ${ }^{34}$ se demolían los añadidos exteriores y las tabiquerías, las máquinas de cubierta y los elementos en mal estado, recuperando la espacialidad original. La urbanización de las zonas exteriores se añade para cumplir las condiciones de evacuación y como ampliación de zonas estanciales y de descanso de los trabajadores. Para cumplir con la normativa de accesibilidad,

31. Usos característicos: ferias o eventos, cultural, deportivo, la restauración y la enseñanza. Autorizados temporalmente: seguridad, protección ciudadana, administración de justicia u otros similares.

32. Programa operativo del Complejo Municipal de Impulso del Asociacionismo, 7 de diciembre de 2017.

33. Catálogo de Edificios, Ficha n. ${ }^{\circ} 41$. Plan Especial “Feria del Campo" AOE 00 04. Madrid: Ayuntamiento de Madrid, 2006.

34. Proyecto de rehabilitación de edificios gestionados por Madrid Destino en los recintos feriales de la Casa de Campo. Complejo Municipal de Impulso del Asociacionismo. Ed. Pabellón de Exposiciones. INCOSA S. A. Arquitecto: David Espigado. Madrid, noviembre de 2018. 


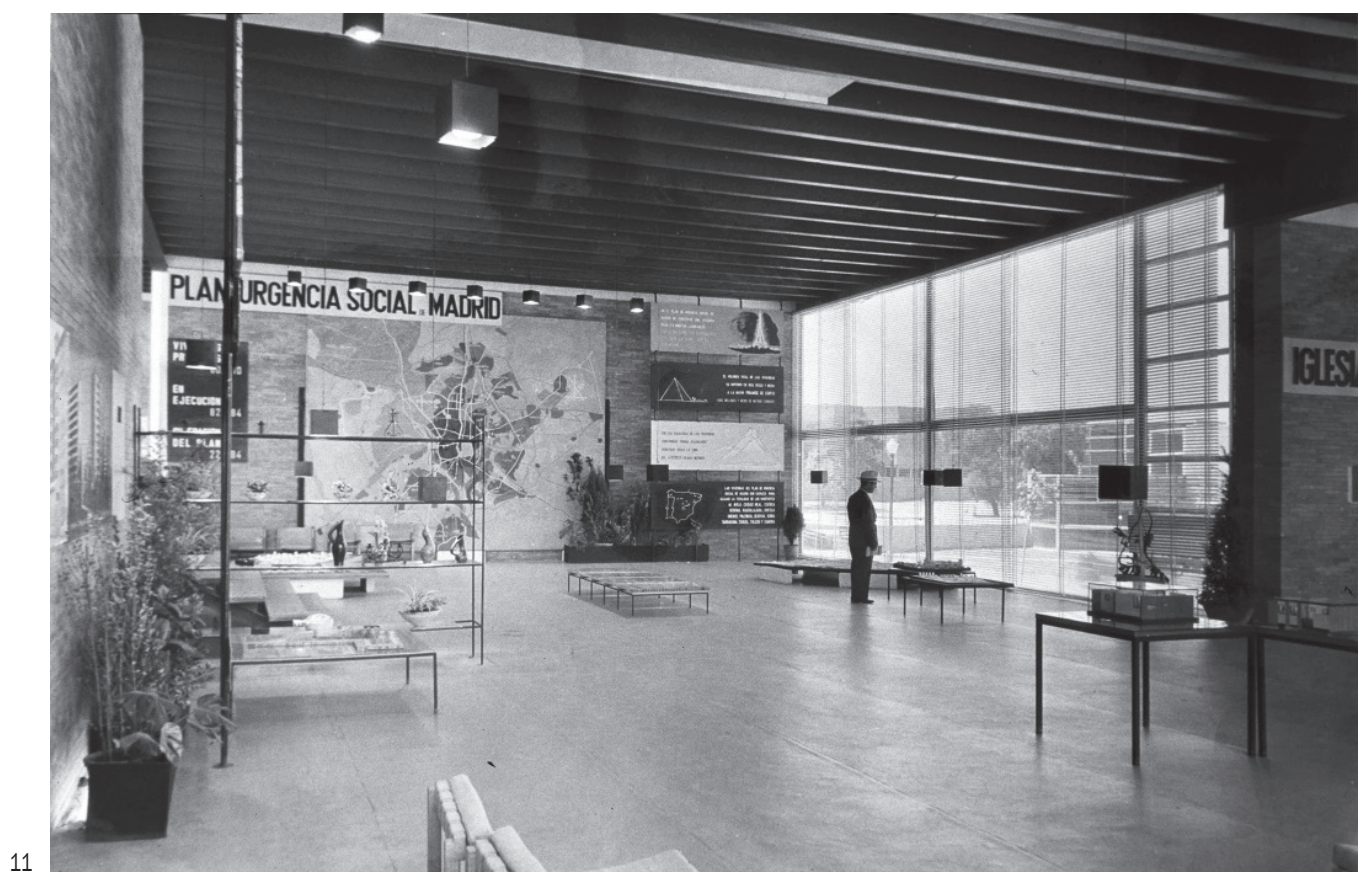

se sitúa un elevador entre la nave de entrada y la $2{ }^{a}$, que comunica el sótano, donde están los aseos de personal y entre las naves 3. ${ }^{\mathrm{a}}$ y 4 . $^{\mathrm{a}}$ una plataforma elevadora como solución más discreta.

Los despachos, zonas de trabajo y de reuniones independizadas añadidos cuentan con un núcleo de servicios y una sala de formación abierta, manteniendo la diafanidad interior mediante la inserción de unos módulos prefabricados de vidrio ${ }^{35}$. Estos podrían retirarse fácilmente para garantizar la reversibilidad al espacio expositivo original, lográndose, además, ampliar los espacios requeridos sin aumentar la edificabilidad. Todas las instalaciones debían hacerse nuevas, lo que supone otra ampliación y adecuación del pabellón a los estándares actuales. Se situó la central de producción de clima en el pabellón de Valencia que servía a las Unidades de Tratamiento de Aire (UTA) de cada pabellón del Campus, servidumbre que puede condicionar negativamente su uso independiente en el futuro.

La estructura original se conservaba bien sin problemas de corrosión importantes, aunque se detectó una flecha excesiva, claramente perceptible, en las viguetas IPN 240 mm debida a su cálculo estricto ${ }^{36}$. Práctica frecuente en los pabellones de la feria, que se hacían con presupuestos y licitaciones a la baja ${ }^{37}$.

Por su mal estado, se proponía demoler las bovedillas de hormigón y sustituirlas por unas aligeradas reduciendo la flecha. En las catas se detectó una estructura de soportes y vigas ocultas en los muros capuchinos que recibían las viguetas de cubierta simulando el apoyo de estas en el muro de ladrillo, solución que vimos en el anteproyecto. Esta estructura se rigidiza en obra mediante arrostramientos diagonales. Los muros capuchinos, sin atado entre las hojas, sufrían graves problemas de estabilidad debido a su altura y al pandeo de los soportes interiores, por lo que se plantea su sustitución respetando las características del ladrillo original sin llaga y disponiendo las necesarias llaves de atado (figura 13).

En obra, se perfeccionaron las decisiones del proyecto contando con la asesoría de un experto en el pabellón y la obra de Francisco Cabrero para respetar al máximo sus valores arquitectónicos ${ }^{38}$. Se realizaron pruebas

35. Los grandes en la dirección longitudinal miden 3,45 × 9,90 m, los pequeños en transversal 3,45 × $6 \mathrm{~m}$, y 2,70 m de altura.

36. Según la memoria del proyecto de rehabilitación, se cumplen los estados límites últimos con una tensión del 98 \% de la admisible. Las flechas máximas debidas a la totalidad de las cargas son de $105 \mathrm{~mm}$, superando los límites admisibles (L/250), pero no comprometen la seguridad de la estructura, que ha aguantado 60 años perfectamente. El aligeramiento de la cubierta permite incorporar la sobrecarga de mantenimiento y reducir la flecha permanente.

37. Testimonio de Jaime Ruiz. Ver: COCA, José de, op. cit. supra, nota 2, p. 74

38. Convenio de Asistencia Técnica suscrito entre la Fundación General de la Universidad Politécnica y la empresa Adjudicataria de las obras Ferrovial Agromán S. A. Hubiera sido deseable incorporar la asistencia ya durante la redacción del proyecto. 
12. Pabellón de Exposiciones. Rehabilitación 2020. 1 Vestíbulo. 2 Asociaciones y federaciones. 3 Cajas de vidrio. 4 Núcleo de servicios. 5 Formación. 6 Espacio exterior. 7 Acceso instalaciones, 2020. 13. Estructura del pabellón de Exposiciones durante las obras de rehabilitación. Fotografía del autor. 14. Pabellón de Exposiciones. Rehabilitación 2020. Ampliación de usos: 1 Cajas de vidrio. 2 Elevador. 3 Núcleo de escaleras. 4 Escaleras y plataforma elevadora. 5 Servicios. 6 Zona exterior de estancia e itinerario accesible. 7 Acceso instalaciones. Sección, planta y detalles muro cortina y cajas de vidrio.
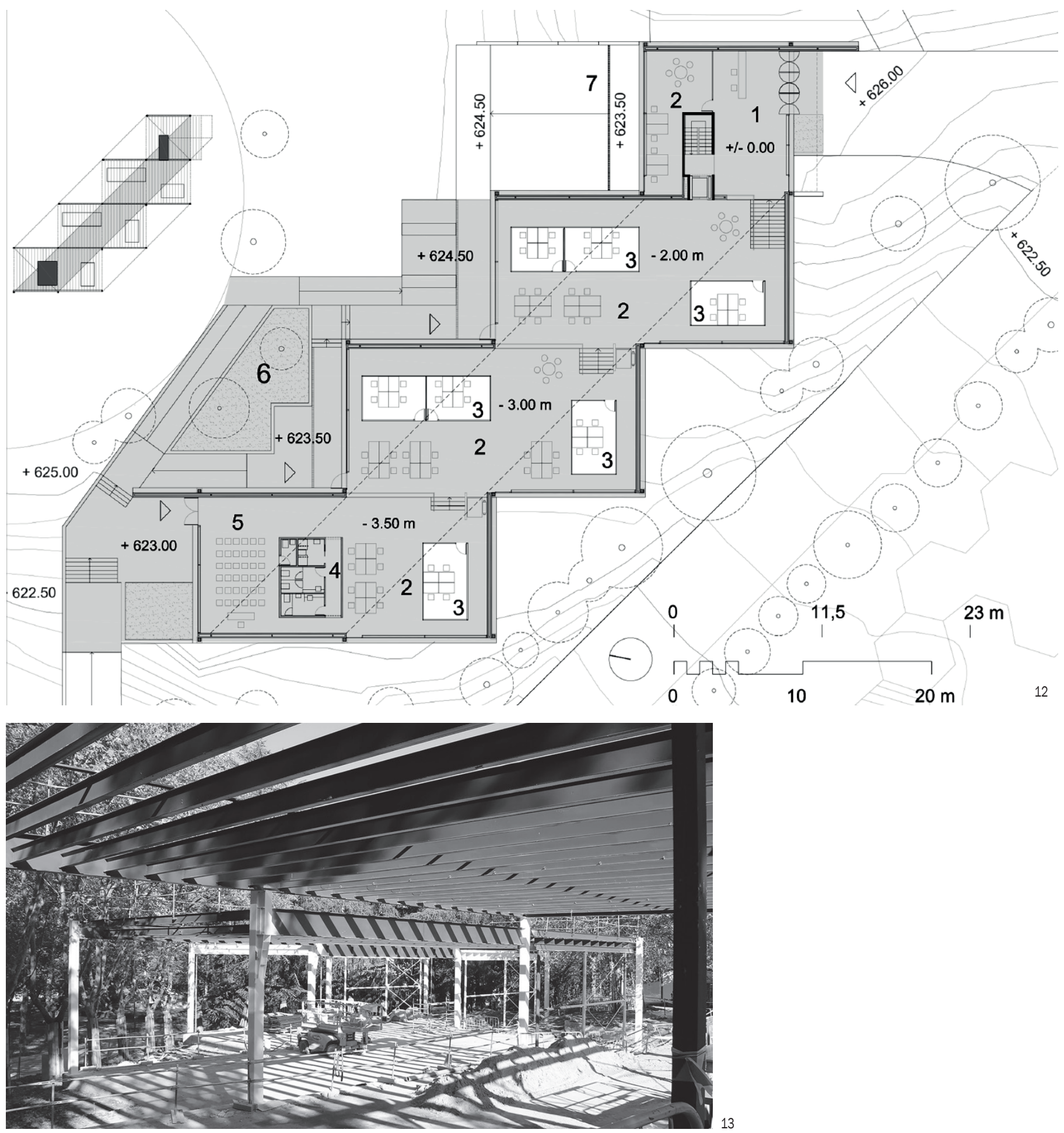


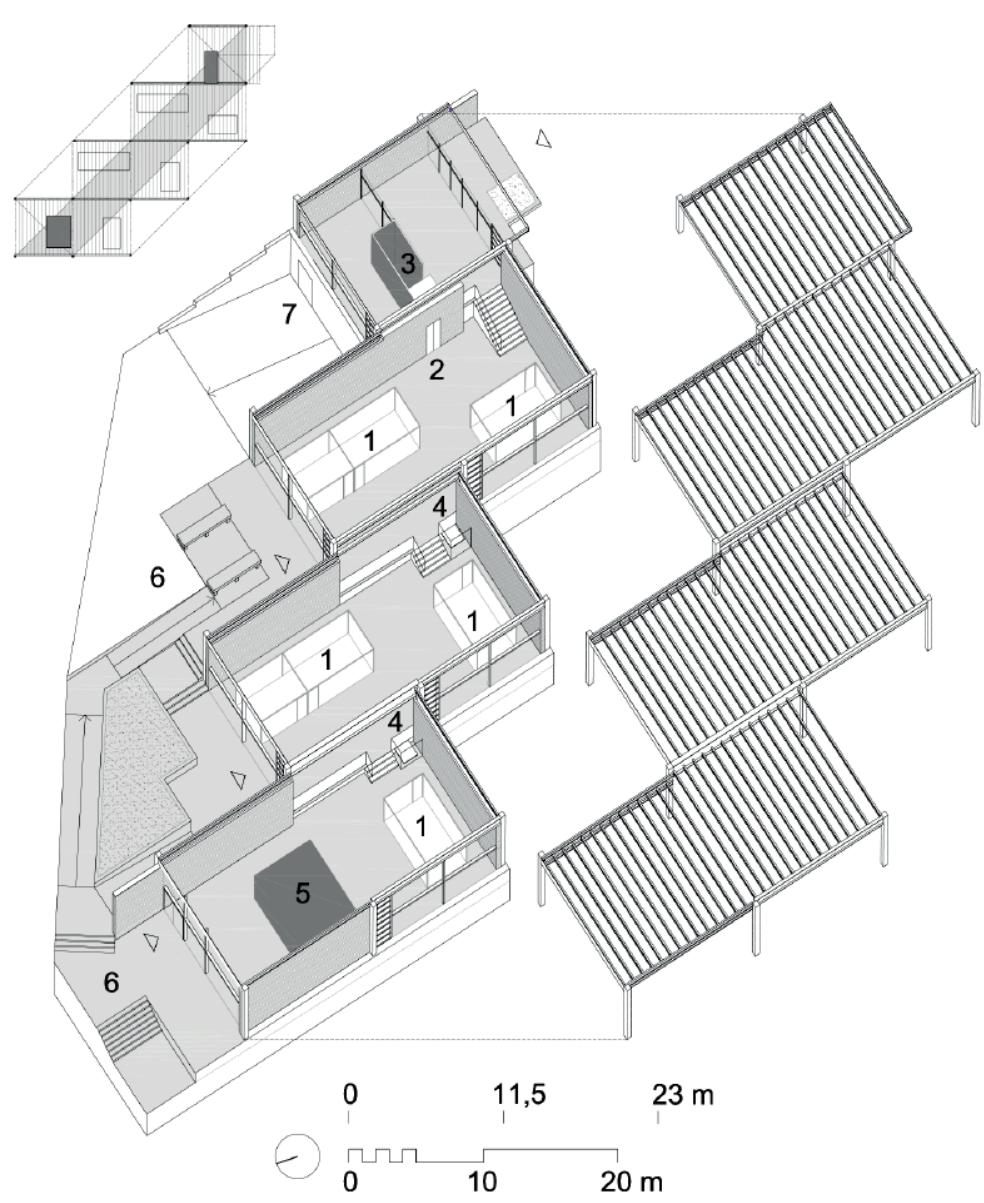

confirmando el color rojo original de la estructura utilizado por Francisco Cabrero ${ }^{39}$. La cubierta, por ligereza y facilidad de montaje, se realiza mediante una solución tipo Deck apoyando la chapa plegada de acero galvanizado en los IPN $240 \mathrm{~mm}$ mediante unos separadores que crean una cámara que permite ocultar la instalación eléctrica y alojar las planchas de escayola de $30 \mathrm{~mm}$ de espesor que formaban el cielo raso blanco imitando las bovedillas originales. En la cara superior se dispuso un aislante de poliisocianurato (PIR) de $50 \mathrm{~mm}$ y doble lámina asfáltica con autoprotección mineral con canaletas de recogida longitudinales situadas entre cada nave.

Otro tema difícil fue minimizar el impacto visual de los conductos de climatización, imprescindibles para el nuevo uso previsto ${ }^{40}$. Tomando como referencia los criterios

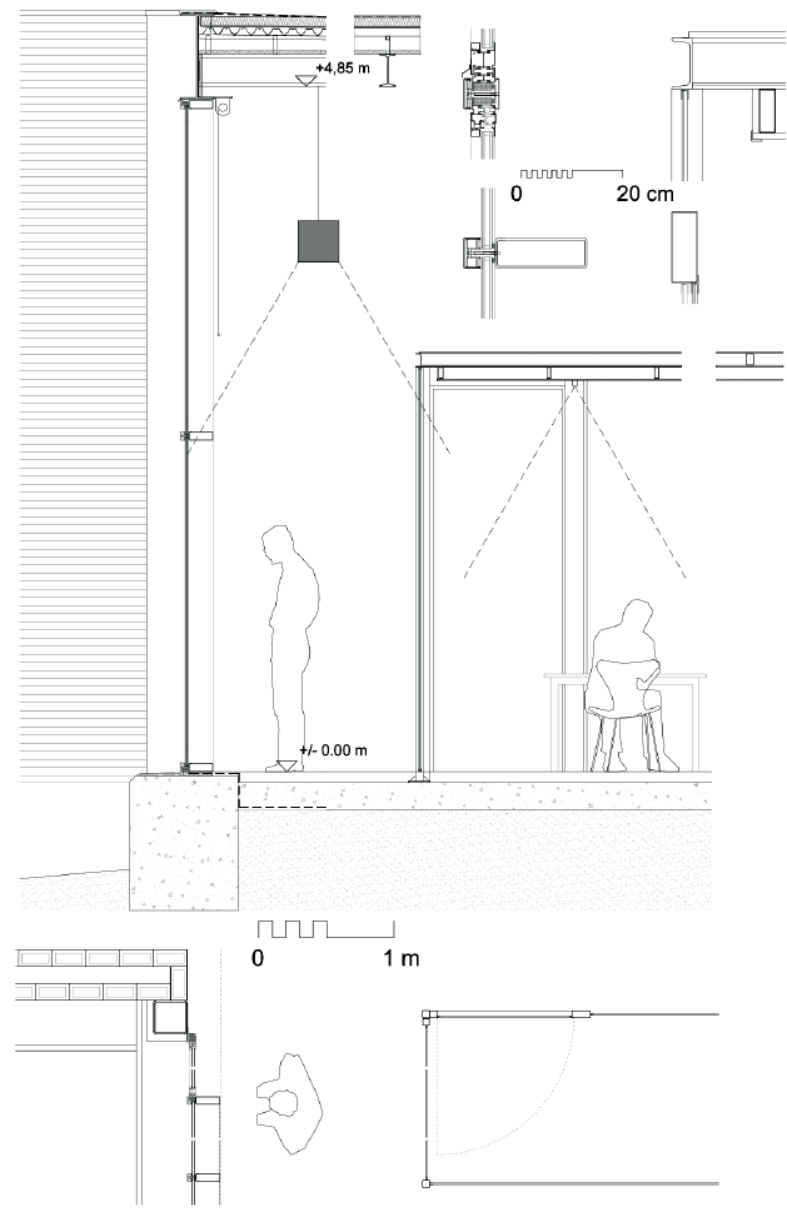

empleados por Cabrero, Ruiz y Labiano en el pabellón de Cristal, se instalaron con su aspecto en acero galvanizado tratando de ordenar al máximo la instalación. Finalmente, en lo referente a la decoración interior, se recuperó el acabado original del hormigón de las bancadas.

En obra se advirtió que las cápsulas de vidrio prefabricadas alteraban la estética del pabellón, no siendo fácilmente reversibles. Se realizaron in situ con una estructura mínima de tubo de acero de $60.60 .2 \mathrm{~mm}$, vigas perimetrales y viguetas UPN $100 \mathrm{~mm}$, todo lacado en rojo, con carpinterías de aluminio y grandes superficies de vidrio laminar de $5+5 \mathrm{~mm}$, emulando la lógica y estética del edificio con "unos pabellones dentro del pabellón" colocados donde interferirían menos con la visual entre las naves $^{41}$ (figuras 12 y 14).

39. RAL CLASIC 3011 “Rojo pardo”. Código RGB Rojo: 121 (47 \%), Verde: 36 (14 \%), Azul: 35 (14 \%).

40. Se estudió llevar los conductos enterrados y utilizar las cámaras del ladrillo para impulsión y retorno. Solución ingeniosa, pero inviable económicamente y técnicamente compleja.

41. En obra se acondicionaron con una unidad individual de intercambio de aire con la sala. 


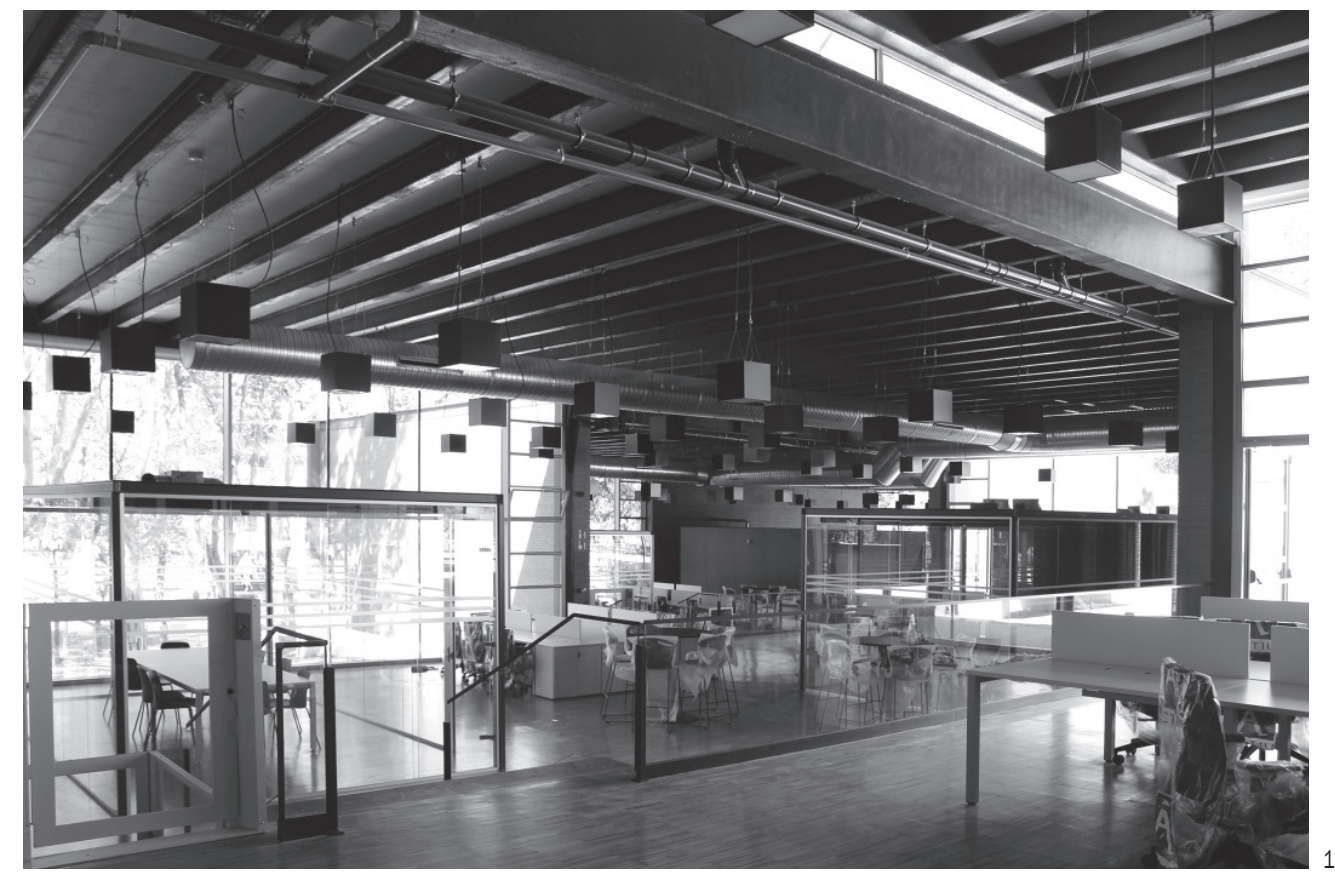

15. Interior con plataforma, escaleras, cabinas de vidrio, luminarias y muros cortina.

Un problema, típico en las rehabilitaciones de arquitecturas modernas, fue minimizar los puentes térmicos debidos a la estructura vista de acero mediante un aislamiento interior de panel rígido pintado de rojo que se fijó al alma de las vigas, siendo prácticamente imperceptible. Los paños de vidrio, reconstituidos ${ }^{42}$ a partir de las fotografías, mantienen las divisiones originales modificando la solución de perfil estructural de acero y fijo de aluminio que implicaría graves problemas de condensación (ver figuras 7 y 14). Se optó por adaptar un perfil comercial de muro cortina de acero de $180 \times 60 \mathrm{~mm}$, lacado en color aluminio natural, con rotura de puente térmico y vidrio de seguridad con cámara aislante ${ }^{43}$. El enorme peso de los vidrios y la facilidad de reposición obligó a introducir entre las lunas unas varillas de acero de métrica 10 para evitar el pandeo, igualando así el perfil vertical y horizontal del muro cortina; despiezando cada hueco con 3 vidrios de $1,60 \times 2,50 \mathrm{~cm}$ de alto.

En cuanto al mobiliario, por cuestiones de ergonomía y salud laboral, se utilizaron muebles actuales en colores blanco y negro que encajasen con la estética de los años 60. Una aportación interesante fue reproducir las luminarias originales, unos cubos negros colgados de la estructura, creando un plano visual minimizando impacto de los conductos de clima. Las escaleras se rehicieron manteniendo la estética original, pero adaptada a las determinaciones que impone el Código Técnico de la Edificación (figura 15).

42. Al no conservarse el elemento original ni los planos, el detalle constructivo "reconstituido" es una mera hipótesis. Ver: MARTíNEZ DíAZ, Ángel; ORTEGA VIDAL, Javier. Investigación y reconstrucción gráfica. En: Actas 13 Congreso Internacional de Expresión Gráfica Arquitectónica. Valencia: Universitat Politécnica de Valencia, 2010, pp. 281-286.

43. Muro cortina de acero zincado y estanqueidad a base de juntas EPDM, con tapeta exterior en acero inoxidable, lacado color aluminio. Acristalamiento doble 8+8.2/16 Ar/6+6.2. Vidrio recocido 2 láminas de butiral, capa de control solar y baja emisividad, cámara de argón de $16 \mathrm{~mm}$ con doble sellado perimetral butilo-silicona UV. Cada vidrio pesa $280 \mathrm{~kg}$. 


\section{AMPLIAR EL USO Y LA VIDA DE LOS EDIFICIOS}

El reconocimiento, la protección y la intervención en las arquitecturas del siglo XX va siendo habitual, aunque seguimos en un contexto de amenaza constante, por lo que queda un largo camino por recorrer. Una cuestión que surge con la intervención en obras emblemáticas, como el edificio de la Bauhaus en Dessau (1925), la villa Savoye en Poissy (1929), la reconstrucción del pabellón de Alemania en Barcelona (1929) o, más recientemente, el Gobierno Civil de Tarragona (1957), por fijar algunos ejemplos, es que, a partir de unos criterios generales consensuados -que difieren de los del patrimonio histórico por el carácter experimental y efímero de estas arquitecturas-, la praxis supone su revisión constante debido, entre otros factores, al uso al que se destina la obra.

En obras como el pabellón de Barcelona o la villa Savoye, el objetivo es su contemplación emulando con su reconstrucción arqueológica, no exenta de debate, el espíritu de la época que las vio nacer, surgiendo la pregunta en villa la Savoye: ¿sigue siendo la misma vivienda después de tantas intervenciones manteniéndola en un presente eterno, negándolas a envejecer? ${ }^{44}$ Otras obras, que conservan su uso original, como el edificio del Bauhaus en Dessau o el Gobierno Civil, tratan de recuperar al máximo posible el bien original, pero con técnicas diferentes de las originales, y edificios como el Bauhaus son objeto de intervenciones planteándose la "'desrestauración"' de fases previas"45.

Los edificios en el campus del IIT han sido objeto de restauraciones de referencia. El Crown Hall (1956), sigue 65 años después como escuela de arquitectura. La restauración de los muros cortina realizada por Krueck+Sexton ${ }^{46}$ parte del estudio de las patologías identificando las causas de la corrosión y la rotura de vidrios. Después de tratar con chorro de arena y repintar la estructura del color negro original, se sustituyeron las lunas originales de $6 \mathrm{~mm}$ por vidrios de seguridad de 9 $\mathrm{mm}$, reemplazando los tapajuntas de sección rectangular por otros trapezoidales para evacuar el agua evitando así la corrosión, sin alterar la estética, pero manteniendo los puentes térmicos obviando los criterios de eficiencia energética en aras de mantener el detalle diseñado por Mies. En la capilla del campus (1952) las intervenciones como el edificio, han sido más modestas, saneando y actualizando la impermeabilización de cubierta con el rediseño del detalle de cornisa y la sustitución de los ladrillos agrietados por los de una pared del edificio Baily Hall, del mismo tipo y época ${ }^{47}$.

Los criterios aplicados en los edificios anteriores, por lo general, se basan en intentar una "reconstrucción a origen" 48 reproduciendo las técnicas originales y sin adecuar los edificios a las exigencias normativas actuales.

En el caso estudiado, estas arquitecturas han sido ampliadas desde otro punto de vista, que es tratar las arquitecturas modernas desde la idea de su reciclaje, donde prevalece la reutilización, y no solo la restauración en sí misma ${ }^{49}$. La propuesta del Campus intenta transmitir a los ciudadanos, mediante un uso compatible, los valores e identidad del patrimonio del siglo $X X^{50}$ no como una restauración rígida, sino como una rehabilitación útil, con nuevos usos, pero respetuosa con los valores originales,

44. MARTÍNEZ MEDINA, Andrés. Las huellas del tiempo de la arquitectura moderna intervenida. En: AAVV. Criterios de intervención en el patrimonio arquitectónico del siglo XX. Conferencia Internacional CAH20thC. Documento de Madrid 2011. Madrid: Ministerio de Cultura, 2011, pp. 395-401.

45. Ver MARKRAF, Monika; OELKER; Simone; SCHWARTING, Andreas. Denkmalplege der Moderne. Konzepte für ein junges Architekturerbe. Wüstenrot Stiftung. Stuttgart: Karl Krämer Verlag, 2011.

46. SEXTON, Mark. Restoration of Crown Hall. En: Ludwig MIES VAN DER ROHE et al., op. cit. supra, nota 25, pp. 65-71.

47. HARBOE, G., op. cit. supra, nota 25, pp. 73-77.

48. MARTÍNEZ MEDINA, Andrés, op. cit. supra, nota 44, p. 398.

49. MARTÍNEZ MONEDERO, Miguel. Reciclaje de arquitecturas vs. restauración arquitectónica, ¿̇herramientas contrapuestas? En: Hábitat y Sociedad. Reciclaje de barrios [en línea]. Sevilla: Universidad de Sevilla, 2012, n. ${ }^{0}$ 5, pp. $23-33$ [consulta: 17-02-2021]. ISSN: 2173-125X Disponible en: https://revistascientificas. us.es/index.php/HyS/article/view/4131 D0I: https://doi.org/10.12795/HabitatySociedad.2012.i5.03

50. Art. 9.2 "Comunicar los valores del patrimonio de forma amplia". En: AAVV. Criterios de intervención en el patrimonio arquitectónico del siǵlo XX, op. cit. supra, nota 44, pp. 23-28. 
y en ese sentido pensamos que el resultado es una arquitectura ampliada. Los criterios generales de intervención, acordes con el documento de Madrid ${ }^{51}$, se han aplicado con sus singularidades en cada edificio. En dos de los edificios de Cabrero, el pabellón de la Obra Sindical, realizado con Pérez Enciso y el del Ministerio de la Vivienda con Ruiz, proyectados con solo tres años de diferencia, pero de muy diferente arquitectura, algunas soluciones añadidas, como el clima, la disposición de barandillas de vidrio para el cumplimiento del Código Técnico o el tratamiento de los suelos con parqué industrial se han homogeneizado dando continuidad a los criterios de intervención en los dos edificios.

La propuesta del Campus es innovadora al introducir un nuevo uso demandado por la sociedad en un recinto originalmente expositivo, pero en el que falta un plan estratégico que coordine este tipo de actuaciones. Evidentemente, el uso expositivo es el principal, los pabellones fueron pensados para eso y el valor y la necesidad de la preservación de su arquitectura moderna son incuestionables. El reciclaje reversible de estas arquitecturas, dándoles una segunda oportunidad con los usos compatibles del Plan, no impide la rehabilitación de otros pabellones, como el de Hexágonos o el de Convenciones, que junto con el de Cristal seguirán manteniendo su uso expositivo enriqueciendo la funcionalidad del recinto.

En cuanto al detalle de las intervenciones, en el pabellón de Exposiciones se intenta resolver la difícil ecuación de preservar un edificio de enorme valor arquitectónico y cultural, pero sin documentación, pérdida de elementos originales, mal ejecutado y con graves patologías por la economía y el carácter efímero de la Feria. De hecho, el único elemento original que se ha podido mantener y sanear es la estructura de bancales de hormigón y entramados espaciales de acero. Da que pensar que lo único que pervive es el elemento protagonista de la "edad del hierro", el resto es "aderezo" sobre el elemento esencial que pauta y configura el espacio. Cabrero probablemente estaría satisfecho, las fotografías inéditas de la estructura libre de cerramiento son un reflejo de la modernidad, del fin "utilitario-social" y de la "belleza" que debe acompañar a la arquitectura, en origen y ampliada.r

Este artículo recoge los resultados del Proyecto de Investigación dirigido por el autor, Ref: 43824812045 "Asistencia Técnica para el pabellón de Exposiciones", en el marco del artículo 83 de la LOU, suscrito entre la Fundación General de la Universidad Politécnica de Madrid y la entidad financiadora Ferrovial Construcción S. A.

51. Ídem. 


\section{Bibliografía citada}

AAVV. Criterios de intervención en el patrimonio arquitectónico del siglo XX. Conferencia Internacional CAH2OthC. Documento de Madrid 2011. Madrid: Ministerio de Cultura, 2011.

CASTRO, Carmen. Entrevista con Francisco de Asís Cabrero. En: Arquitectura. Madrid: COAM, 1973, n. ${ }^{\circ}$ 172, p. 6.

CABRERO, Francisco de Asís. Francisco de Asís Cabrero. Título 1942. En: Arquitectura. Madrid: COAM, 1964, n. ${ }^{\circ}$ 61, p. 16.

CABRERO, Francisco de Asís. Los cuatro libros de la Arquitectura. Madrid: COAM, 1992.

CLIMENT, Javier. Francisco Cabrero, arquitecto: 1939-1978. Madrid: Xarait, 1979.

COCA, José de. El recinto ferial de la Casa de Campo de Madrid (1950-1975). Directores: José María Mercé y Javier Ortega. Tesis doctoral. Universidad Politécnica de Madrid, Departamento de Proyectos Arquitectónicos, 2013.

MARTÍNEZ DÍAZ, Ángel; ORTEGA VIDAL, Javier. Investigación y reconstrucción gráfica. Actas 13 Congreso Internacional de Expresión Gráfica Arquitectónica. Valencia: Universitat Politécnica de Valencia, 2010, pp. 281-286.

MARTÍNEZ MEDINA, Andrés. Las huellas del tiempo de la arquitectura moderna intervenida. En: AAVV. Criterios de intervención en el patrimonio arquitectónico del siglo XX. Conferencia Internacional CAH20thC. Documento de Madrid 2011. Madrid: Ministerio de Cultura, 2011.

MARTÍNEZ MONEDERO, Miguel. Reciclaje de arquitecturas vs. restauración arquitectónica ¿herramientas contrapuestas? En: Habitat y sociedad. Reciclaje de Barrios [en línea] Sevilla: Universidad de Sevilla, 2012, n. ${ }^{\circ}$ 5, pp. 23-33 [consulta: 17-02-2021]. ISSN: 2173-125X. Disponible en: https:// revistascientificas.us.es/index.php/HyS/article/view/4131 DOI: https://doi.org/10.12795/HabitatySociedad.2012.i5.03

MATA, Sara de la; SOBEJANO, Enrique. Entrevista a Francisco de Asís Cabrero. En: Arquitectura. Madrid: COAM, 1987, n. ${ }^{\circ} 267$, pp. $110-115$.

MIES VAN DER ROHE, Ludwig, et al. The Heritage of Mies. Eindhoven: Eindhoven Docomomo International Secretariat, 2017, n. ${ }^{0} 56$ (2017/1).

Pabellón del Ministerio de la Vivienda. En: Arquitectura. Madrid: COAM, julio 1959, n. ${ }^{0}$ 7, pp. 16-19.

RUIZ CABRERO, Gabriel. Vida y obra de Asís Cabrero. En: AAVV, Francisco de Asís Cabrero. Legado 02. Madrid: Fundación COAM, 2007, pp.10-109.

SÁNCHEZ DE LA CHICA, Juan Manuel. Materia, material y aparejo en la arquitectura de Francisco de Asís Cabrero. Director: Gabriel Ruiz Cabrero. Tesis doctoral. ETS Arquitectura, Universidad de Málaga, 2015.

José de Coca Leicher (Madrid, 20 de diciembre de 1965) Escuela Técnica Superior de Arquitectura de Madrid, donde se titula de Arquitecto en 1993 y Doctor Arquitecto con Mención Especial en 2013. Profesor Ayudante Doctor en la ETSAM en el Departamento de Ideación Gráfica Arquitectónica y docente desde 1998. Profesor en la Escuela de Arquitectura de Alcalá en las Áreas de Expresión Gráfica Arquitectónica (2002-2006) y Proyectos Arquitectónicos (2006-2016). Gastprofessor en la Technische Universität Mittelhessen en el Departamento de Arquitectura los semestres de verano de 2014 y 2015 en Proyectos Arquitectónicos y Dibujo. Publica en las revistas indexadas PPA, EGA, Constelaciones y Cuaderno de Notas, colaborando de revisor. Capítulos de libros en Springer, EGA y Pioneros de la FAS. Asesor del Ayuntamiento de Madrid y Asistencia Técnica en las obras de rehabilitación de los pabellones del recinto Ferial de la Casa de Campo de Madrid, tema de su tesis publicada en Ed. Asimétricas. 


\section{Autor imagen y fuente bibliográfica de procedencia}

Información facilitada por los autores de los artículos:

página 19, 1. WOODBRIDGE, Kenneth. Princely Gardens. Nueva York: Rizzoli, 1986; página 21, 2. ALPHAND, J. C. Adolphe. Les Promenades de Paris. Princeton: Princeton Architectural Press, 1984, p. 199; página 22, 3. AURICOSTE, Isabelle; TONKA, Hubert. Parc Ville Villette. Seyssel: Champ Vallon, 1987; páginas 23,24 y 25, 4, 5 y 6. Fotografías de Ramón R. Llera; páginas 26 y 27, 7 y 8 . BASSO PERESSUT, Luca; CALIARI, Pier Federico, eds. Piranesi Prix de Rome. Progetti per la nuova Via dei Fori Imperiali. Roma: Aión Edizioni, 2017; página 28, 9. LAB/PAP Laboratorio de Paisaje Arquitectónico, Patrimonial y Cultural; páginas 28 y 29, 10, 11 y 12 . BASSO PERESSUT, Luca; CALIARI, Pier Federico, eds. Piranesi Prix de Rome. Progetti per la nuova Via dei Fori Imperiali. Roma: Aión Edizioni, 2017; páginas 30 y 31 ,13, 14 y 15. ÁLVAREZ, Darío; IGLESIA, Miguel Ángel de la. Modelos de paisajes patrimoniales. Estrategias de protección e intervención arquitectónica. Valladolid: LAB/PAP y Departamento de Teoría de la Arquitectura y Proyectos Arquitectónicos, Universidad de Valladolid, 2017; página 36, 1. Philippe BOURDIN, “Le sacre de Louis XV", Histoire par l'image [en línea], consultado el 20 de agosto de 2020. Disponible en: http://histoire-image.org/fr/etudes/sacre-louis-xv; página 37, 2. Archivo de José lgnacio Linazasoro Rodríguez; página 38, 3. JAKOVUEVIC, Nada; CULOT, Maurice, dirs. Places et monuments. Bruselas: Mardaga, 1984; página 40, 4. RIGAUD, Olivier. Les plans anciens de Reims, 16001825. Dossier iconographique. Reims: RHA, 1991; página 41, 5. Biblioteca Municipal de Reims [en línea], consultado el 20 de agosto de 2020. Disponible en: https:// commons.wikimedia.org/wiki/File:Rue_Libergier_Cathédrale_Reims_Varin_30131.jpg; página 42, 6. Revista UR, 1985, n. ${ }^{\circ}$ 2; página 43, 44, 46, 47, 48, 49, 50, 7, 8, 9 , 10, 11, 12, 13, 14, 15. Archivo de José Ignacio Linazasoro Rodríguez; página 54, 1. Eisenman Architects Archives; página 55, 2. DR2004:0360:003 Cedric Price fonds Canadian Centre for Architecture; página 56, 3. DR2004:0355:007. Cedric Price fonds Canadian Centre for Architecture; página 57, 4. DR2004:0355:006. Cedric Price fonds. Canadian Centre for Architecture; página 59, 5. ARCH267828. IFCCA Prize Competition for the Design of Cities fonds Canadian Centre for Architecture; página 59, 6. Dominio público.; página 60, 7. Archivo Lacaton \& Vassal; página 61, 8. Fotomontaje de los autores a partir de Google Street View (2018); página 62, 9. Fotografía de Philippe Ruault:; página 63, 10. Muestra de la web del proyecto con el reportaje fotográfico de Philippe Ruault.; página 64,11 . Archivo Lacaton \& Vassal.; página 65,12 . Vista aérea en Google Maps (2018):; página 65, 13. Vista obtenida en Google Street View (2018); página 66, 14. Archivo Lacaton \& Vassal, 2004.; página 67, 15. Vista obtenida a través de Google Street View (2018); páginas 74 y 75, 1, 2 y 3. @ Krijn de Koning, cortesía del artista; páginas 76 y 77, 4, 5 y 6 . @ Jessica Stockholder, cortesía de la artista y de Mitchell-Innes \& Nash, Nueva York; páginas ,78 y 79, 7, 8 y 9. @ Katharina Grosse, VEGAP, Sevilla, 2021; páginas 82 y 83, 10 , 11 y 12. Heather Day website. Disponible en: https://heatherday.com/downtownoakland; página 90, 1. Universidad de Zaragoza. Unidad Técnica de Construcciones.; página 91, 2. Planimetría redibujada por Clara Dobón Ricarte.; página 92, 3. Planos, redibujada por Clara Dobón Ricarte. Fotografías cortesía de la Facultad de Derecho de la Universidad de Zaragoza.; página 93, 4. Google Maps.; página 94, 5. Fotografías de los autores.; página 95, 6. Proyecto de Ejecución, Ampliación y Reforma de la Facultad de Derecho (Zaragoza). Fuente: Universidad de Zaragoza. Unidad Técnica de Construcciones.; páginas 96 y 98, 7, 8. Planos y axonometría dibujados por Clara Dobón Ricarte; páginas 99 y 101, 9, 10, 11. Fotografías de los autores.; página 102, 12. Fotografía (izquierda) de los autores y fotografía (derecha) cortesía de la Facultad de Derecho de la Universidad de Zaragoza.; página 102, 13. Fotografías de los autores.; página 103, 14. Fotografía cortesía de Javier López Sánchez, decano de la Facultad de Derecho de la Universidad de Zaragoza.; página 104, 15. Proyecto de Ejecución, Ampliación y Reforma de la Facultad de Derecho (Zaragoza). Fuente: Universidad de Zaragoza. Unidad Técnica de Construcciones; página 108, 1. Dibujos del autor; página 109, 2. Ayuntamiento de Madrid; página 110 y 112, 3 y 4. Ayuntamiento de Madrid; página 113 y 114, 5 y 6 . Archivo Cabrero; página 115, 7. Reconstitución gráfica del autor; página 116, 8. Legado 2 Fundación COAM; página 117, 9. Dibujos del autor; página 118, 10. Arquitectura, 1959, n. 7 y Francisco de Asís Cabrero y Jaime Ruiz; página 119, 11. Archivo Cabrero; página 120, 12. Dibujos del autor; página 120, 13. Fotografía del autor; página 121, 14. Dibujos del autor; página 122, 15. Fotografía del autor. 
ARQUITECTURAS AMPLIADAS. EL PABELLÓN DE EXPOSICIONES EN LA CASA DE CAMPO DE MADRID EXPANDED ARCHITECTURES. THE EXHIBITION PAVILION AT THE CASA DE CAMPO IN MADRID José de Coca Leicher (https://orcid.org/0000-0002-0950-4163)

p.107 "I really think that the population and the anthropological development curves can be overlapped on a continuous curve of the development of architecture, or rather culture as a whole, because architecture is the camera of culture: it has got a lot of science and a lot of sense of living..."1.

The rehabilitation of the Exhibition Pavilion, a unique sample of modern Spanish architecture, shows the challenges and difficulties within the practice of recovering and adapting current buildings. In this case, the original exhibition space, as well as the interest on the building solution, must fulfill new requirements that force the building updating and expanding. The analysed solution responds to these strategies by inserting little architectures, facilities to meet the climate control and accessibility requirements and to layout the outdoor spaces. Other three buildings of different value and rank of protection are involved in the Campus Asociativo de Madrid action. This action is implemented at Casa de Campo Fairgrounds in Madrid, a protected venue as an urban area whose history and conditions require a prior review (fig. 1).

\section{ORIGIN, CONTEXT AND PRESENT SITUATION OF THE ENCLOSURE}

The Casa de Campo de Madrid exhibition centre goes back to the country fairs held between 1950 and 1975 to improve and modernize agriculture and livestock. Architects Francisco de Asís Cabrero and Jaime Ruiz designed an original 15Ha venue, where the 1st National Country Fair was inaugurated in 1950. The construction of the enclosure was determined by stands of vaults and brick arches, a circular welcoming square and a modern lookout tower facing Madrid skyline. Nowadays only a few buildings and the pine forest facing the Manzanares river are preserved².

The 1st International Country Fair was inaugurated in 1953. Cabrero and Ruiz also designed the second 55Ha

p.109 enclosure which expanded the original one onto the West and a beautiful landscape layout with tree-lined avenues and plots where the regional pavilions were located. The "pioneers' buildings" stood out -Ciudad Real Pavilion by Miguel Fisac; Jaén Pavilion by José Luis Romaní and Pontevedra Pavilion by Alejandro de la Sota-, all of them lost modern architectures, inspired in the Spanish folk culture.

The enclosure comprises other representative, protected and well-preserved pavilions by Cabrero and Ruiz as the Ministerio de la Vivienda (Ministry of Housing) -subject of our research-, the Obra Sindical del Hogar (Governmental Housing Institution), the Palacio de la Agricultura (Agriculture Palace) and the International pavilion (fig. 1 and 3). In 1977, when the Country Fairs came to an end, 123 buildings were registered ${ }^{3}$ and a year later, the Dirección de Instalaciones Feriales (Fair Facilities Division) was established. In 1980 the Patronato Municipal (City Board) for the use of facilities in Feria del Campo enclosure was founded ${ }^{4}$.

The precedent urban planning information ${ }^{5}$ to the 1985 democratic Plan General de Ordenación (General Urban Planning) consisted of an initial catalogue and photo inventory. In order to follow the continuity plan in the Casa de Campo, "sponging works" were carried out by demolishing the original vaults belonging to the first country fair. In 2001, due to the municipal board paralysis and the abandonment of the enclosure, the communal company Campo de las Naciones was founded, giving rise to the special plan "Feria del Campo", definitively approved in 2006.

In 2006 Madrid Espacios y Congresos (Madrid Event \& Congress company) was founded with the purpose of dynamizing the area and trying to execute the Special Plan. The catering section at the regional pavilions and the exhibition activity at the representative pavilions were launched as their congress activity was very diminished by the

p.111 transfer of Feria de Madrid (IFEMA) to Juan Carlos I site. In 2013, after the events at Madrid Arena ${ }^{6}$, the fair district was absorbed by Madrid Destino Cultura Turismo y Negocio, the current managing company.

45 years later, the original layout is preserved, some of it altered, and 40 percent of the original buildings exists. Save for the well-known Pabellón de Cristal (Crystal Pavilion) and the former International Pavilion (current Convention Centre), both of them belonging to level 1 Singular Building protection classes which have been restored and maintain the exhibition activity, many symbolic buildings at the core of the enclosure are now occupied by the 112 emergency services or the local police headquarters (former Pipa Pavilion and Agriculture Palace) according to the plan called "Ciudad de las Emergencias"7. Many pavilions, in the absence of a driving plan to implement a suitable public use, host inappropriate uses or they are just empty.

REHABILITATION STRATEGIES: THE SPECIAL PLAN "FERIA DEL CAMPO" AND THE CAMPUS ASOCIATIVO DE MADRID The Special Plan ${ }^{8}$ stood up for the specializing of the site considering its own identity, the intensive use of the historical park for additional exhibitions apart from IFEMA and the compatibility with the facilities and the cultural and leisure activities $^{9}$. The plan focused on remodelling the pavilions, on the promotion of pedestrian spaces and itineraries, on the decrease of the road traffic and on the addition of the Madrid Río initiative linked to the historical park in an effort to involve neighbouring districts.

The catalogue comprises buildings and architectural elements, fixing their state of preservation and the degree and level of protection for the 38 pavilions. On each catalogue file, the original building plan and description are 
provided, as well as the outstanding features, the best quality parts, the expansions to be removed and the items to be restored ${ }^{10}$. Four pavilions were Singular Grade Level 1 graded for their high architectural and referential importance. Nine pavilions were Integral Grade Level 1 protected and other 25 pavilions were Structural Grade Level 2 classified. Lastly, eleven items belonging to disappeared pavilions and the "constructivist" streetlights, designed by Cabrero for the second Fair ${ }^{11}$ were protected (figure 3).

The Campus Asociativo de Madrid ${ }^{12}$ Project was promoted during the last municipal government ${ }^{13}$. It displayed a dual aim: recover the site buildings for the sake of the 20th century cultural heritage and provide the city with facilities. Four pavilions were selected, including our Exposiciones pavilion (Exhibition Hall). They had not been used for years, with the consequent functional and patrimonial loss.

The Icona II pavilion, built by Eduardo Baselga in 1957 and covered with attractive concrete waves that hosted the Forest Department, was Structural Grade Level 2 classified. The Icona I Pavilion, by Francisco Cabrero and Felipe Pérez Enciso, hosted the Obra Sindical del Hogar (Trade Union Housing Department). This building, inspired by Mies van der Rohe's Barcelona Pavilion, displays a restored ceramic mural by Amadeo Gabino and Manuel Suárez Molezún and is Integral Grade Level 1 classified. The regionalistic Valencia VII Pavilion is Structural Grade Level 2 p.112 classified. Both buildings were built in 1959.

The Exhibition Pavilion, the main building in the campus, former Ministry of Housing, was designed by Francisco Cabrero and Jaime Ruiz in 1959. It is Integral Grade Level 1 classified, the highest degree of protection. It is a highly important building for its architectural value and it implies an essential landmark in Francisco Cabrero's professional career and the $20^{\text {th }}$ century modern Madrilenan architecture (fig. 2 and 3).

The Campus (functional programme) planning was laid out following the architectural styles, the functional suitability and the permitted construction works ${ }^{14}$. Icona I was meant for exhibitions and library as there is an open hall in it. The less valueable but more functional Valencia Pavilion was meant for workshops, coworking facilities, community management and meeting areas. Icona II Pavilion was meant for cultural facilities, a stage and a cafeteria. Lastly, the Exposiciones Pavilion, the most representative building, was used for hosting the Federations and for working and management areas so the extraordinary spatiality and the partitioned use were shared (fig. 4).

\section{MINISTRY OF HOUSING PAVILION. THE BEGINNING OF THE "IRON AGE"}

The Ministry Pavilion is the first of an array of works by Cabrero, in collaboration with Jaime Ruiz, between late 50's and mid-60's where the expressive language is reduced to some scarce materials. Cabrero admits the inspiration in Mies van der Rohe's works on the Illinois Institute of Technology (ITT) campus, which he visited in 1956.

In an interview where he also considers the Neoplasticism origin in his work ${ }^{15}$, he defines it as a mix of a plan breakdown, the cubic shape and the orthogonal order to cover the "three architectural imaginations": space, materiality and layout ${ }^{16}$

This thought, based on the abstract nature of Neoplasticism, approaches him to the work of the architect and artist p.113 Max Bill, an admired icon who reaches the inclusion of all arts, embodied in a particular concept or "concret art" in his works, on which Cabrero bases his idea of constructivism (figure 5).

In this stage, ironically defined as the "iron age", Cabrero reduces his architectural language to the properties of steel and the blend of industrial sections. He submits architecture to the language of steel grids, studying the junctures and relying on the material slenderness for him to build enormous lintels ${ }^{17}$. Therefore, the different gratings to cover large openings and panes generate the space with ideal materials in terms of their function and their aesthetics: brick and glass.

Cabrero thinks that the "trascendental" characteristic of $20^{\text {th }}$-century architecture is its "utilitary and social" meaning. The building should provide the "programmed life", which means a perfect social order by the means of "experimented and continuous" artistic forms. ${ }^{18}$ He defines "useful" as something profitable, convenient and advantageous, comprehended in the word "functional". He associates the content to the solution of the problem and the container to the appropriate performance of both building mechanics and structure.

Each programme is associated to a certain building and structural solution at its own mechanics and instruments, which will solve both indoor and outdoor spaces in a building by its own artistic expression. Following Max Bill's definition of "particular art", Cabrero names "constructivism" the word that covers instruments, structural logics, modulation and materials. This item talks about the project own logical decisions, visible in the finished building and being reached by the main idea of activating the artistic abilities of space and matter. The classic ideal remains a proportion in the relationship among parts, adding the central perspective or the foreshortening from a modern dynamic vision based on the "three neoplasticism natures" aforementioned.

Architecture is an art whose aim is not other than reaching the "useful beauty". This utilitarian meaning is governed by direct needs a man must fulfill but not in any way and meanwhile architecture must express itself as an art ${ }^{19}$. The 
"constructivist" and experimental condition of architecture is expressed by steel building ${ }^{20}$, a direct and nude concept defined by Cabrero as unornamented architecture ${ }^{21}$ (fig. 6 and 7).

Knowing the material is essential to the architect: "Wood is not really known [...] until you work with it. And the same happens to iron, stone and other materials22. During this period, Cabrero paints iron structures in red, the red in nature: "I tried minium red tones and then different reds in my works. At my house I decided on a brighter red, which p.114 matches with timber. Some people said that was a Japanese colour but I didn't think so. I realise they were right [...]. I think Japanese architecture is essentially constructivist ${ }^{23}$.

The Ministry of Housing is Cabrero's first work where his principles are applied. He had previously used black covered iron at the Escuela Nacional de Hostelería (National Hotel and Restaurant School), built along with Jaime Ruiz at the fairgrounds in 1956 (still existing). He applied it on the facade structure free from any load trying the differentiation programme with different materials and building systems.

After the Ministry Pavilion, Cabrero built several works where he tried the same "constructivist" solution with variations. The newspaper Arriba building and office block on the Paseo de la Castellana (1962) presents a metal grid $11 \times 11$ brick and glass coated office-chambers. A fiber-cement-coated industrial structure of leaning arches is attached at the rear. Cabrero settles cantilevered pillars and metallic beams over the facade, brick, glass, aluminium or wooden walls and an aluminium roof in his own concrete-founded residence Puerta de Hierro II (1962).

\section{THE ORIGINAL PAVILION PROJECT}

There is a preliminary Project whose design consists of an open $30 \times 15 \mathrm{~m}$ plan and $5 \mathrm{~m}$ height space ${ }^{24}$ and where the close relationship with the ITT Campus, especially with the recently restored Carr Chapel is patent ${ }^{25}$. The inner rectangle plan is limited by a brick and glass-aluminium "L" shape walls. The steel beam framework that leans out from the roof and shapes the interior lays on a brick wall (as in the Carr Chapel) on one side and on the floor on the other side by the use of "L" shaped semiarches with a wall bracket section which is used in other pavilions in the Fairgrounds (fig. 8).

p.115 The final Pavilion, whose plans are not preserved ${ }^{26}$, is adapted to a new $6 \mathrm{~m}$ unevenness lot perpendicular along the street. Opposite to the Pavilion the well-known Brussels Pavilion is built, and an interesting dialogue about them is established. Their architecture shares similar materials, but each of them is based on very different geometrical systems: orthogonal versus hexagonal (see fig. 1).

The Pavilion structure is solved with three and a half halls. The model hall is a $23 \mathrm{~m} \times 11.5 \mathrm{~m}$ plan rectangle ${ }^{27}$,

p.116 double square often used by Cabrero. The interior is again $5 \mathrm{~m}$ height and the preliminary project spatial concept is respected. The halls are stepped down on plan and section to adapt the building to the street alignment and the ground topography. The plan presents a $11.50 \mathrm{~m}$ lag, half its longitude and section, as the main entrance is on the highest point and the steps go gradually down from higher $2.10 \mathrm{~m}$ to lower $0.50 \mathrm{~m}$ (fig. 9).

The halls structurally act as independent side-long-joisted pavilions. They consist of a double corbel "T" $635 \mathrm{~mm}$ side and $250 \mathrm{~mm}$ wing, porches with a $11.50 \mathrm{~m}$ span on $260 \mathrm{~mm}$ side square steel pilars. There is only a $1150 \mathrm{~mm}$ side double "T" corbel beam in the portico between the 3rd and 4th hall. The $11.50 \mathrm{~m}$ openings are covered by IPN $240 \mathrm{~mm}$ joists. As seen in other works by Cabrero, the structure, in order not to double beams and to "prop up" the $1^{1 / 2}$ exposed-brickwork-base walls offers tangent joinings between beams and pilars or "to the fullest" joinings.

There are two published versions of the plan which, compared to the photographs, make us think the built plan belongs to the 1979 monography ${ }^{28}$, where an elevated corridor is planned in the entrance hall from which the view

p.117 covers the whole interior space. However, the remodelling project takes the plan published in Arquitectura magazine as a mode ${ }^{29}$ where the elevated corridor is removed, the stairs are enlarged in the 2 nd hall and the stairs in the third hall are moved to the right following the perimeter (fig. 10).

The Pavilion is founded on concrete basements that shape terraces on the ground. Pilars lay on these basements, on the bearing plates and brick walls. The halls share the lengthwise beams charged by the purlins with a $83 \mathrm{~cm}$ interaxis filled with $12.5 \mathrm{~cm}$ light concrete blocks, a compression layer and light concrete. The roof was an asphalt layer leaning onto small pans in the centre of each hall, from which the horizontal grid comes out, and drainpipes are hidden into the brick wall chambers.

Exposed brick walls and both large $11.5 \mathrm{~m}$ wide $\times 5 \mathrm{~m}$ high glass panes alternate. Save for a sideway $1.25 \mathrm{~m}$ opening windows, vanes were divided by a 2 UPN 160mm red structural cross covering the wall section with simple aluminium and $6 \mathrm{~mm} \times 4.50 \mathrm{~m}$ wide $\times 2.40 \mathrm{~m}$ high glass, following our research. A similar construction with minor glass is found at the Crystal Pavilion side entrance.

The dynamism of the indoor space was astonishing as the overlapping of the halls generates a visual vanishing trace that crosses them as a new diagonal space, either ascending or descending, depending on the observer with a $11.5 \mathrm{~m} \times 5 \mathrm{~m}$ (similar to the brick and glass panes) gap (see fig. 7 and 9). Opposite to the Crystal Pavilion, Cabrero and Ruiz repeated four years later the stepped interior in Bancadas Pavilion with a single inclined plan roof ${ }^{30}$.

p.118 Furniture by Darro consisted of square, tall and short light-tube-structured tables made out of wood, similar to the chairs, items that avoided the interference with the interior spatiality and repeated the idea of an element on legs as it is in the building itself.

We witness the way Cabrero links the content to the programme solution and the continent to the spatial layout. This matter generates scale changes between the brick and glass panes and the furniture. Each wall plays its role as social or intimate space, acting as an information support, a limitation or a visual opening (fig. 11). 


\section{THE REHABILITATION PROJECT}

The "Plan Especial" does not allow a building expansion. The particular uses of the Pavilions are related to the fair activity, so other uses are temporarily allowed, which actually means an expanded use ${ }^{31}$ as the pavilion becomes the head office of federations and associations ${ }^{32}$. In the catalogue file, the most valuable items were "clearness and visual communication among rooms. The rationality of the structure and the doors and windows" and it was established "to remove the Northwest galleries and the inner walls. Remodel roofs and brick covering of vanes. Add glass windows respecting the original plan. Recover original access"33 (fig. 12).

Following the project ${ }^{34}$, the outdoor expansions and inner walls as well as the roof and damaged elements were demolished in order to recover the original spatiality. The outdoor urbanization is added to fulfill the evacuation requirements and the needed social and leisure zones for workers. The accessibility needs are fulfilled by an elevator between the entrance hall and the 2nd hall that goes down to the basement, where the workers' toilets are and an p.119 elevating platform between the 3rd and 4th halls as a more discreet solution.

There is a service division and an open education area within the added offices, working and meeting zones. The inner transparency is kept by premanufactured glass modules ${ }^{35}$, which can be easily removed to maintain the original exhibition space reversible. It achieves, at the same time, the expansion of the needed space without expanding the building. All facilities must be brand-new, so another expansion and adaptation to current standards are needed. The air conditioning central system was settled in Valencia Pavilion, which served the Air Treatment Units (UTA in Spanish) in each pavilion in the campus, a fact that could negatively affect their independent use in the future.

The original structure was well preserved with no important corrosion problems but an excessive deflection was detected, clearly perceptible in IPN 240mm joists, after a strict calculation ${ }^{36}$. This was a frequent policy in the pavilions as tenders and budgets were inclined to diminish ${ }^{37}$.

It was proposed to substitute the damaged concrete jack-arches by lighter ones in order to reduce deflection. During the inspection, it was detected a supporting structure of hidden beams in the isolating brick walls where the joists of the roof were based, as a simulated support in the brick wall, a solution we witnessed in the preliminary project. This structure becomes more rigid thanks to the diagonal bracements. The isolating brick walls showed important stability problems due to their height and the bending of the inner supports. So it was proposed the replacement of those walls respecting the original brick wall with no junctions installing the necessary connectors (fig. 13).

The decisions taken in the Project were improved on site thanks to an expert in both the Pavilion and Francisco Cabrero's works in order to respect their architectural values at the most ${ }^{38}$. Some tests confirmed the original red p.121 colour used by Francisco Cabrero in the structure ${ }^{39}$. The roof is covered with a Deck-type solution, for lightness and ease of assembly, being the galvanized thin layer supported by the IPN 240mm thanks to separating elements that generate a chamber where the electrical wiring is hidden and $30 \mathrm{~mm}$ wide plasterboards are hosted imitating the original jack-arches. On the upper layer a $50 \mathrm{~mm}$ PIR isolating and a double asphaltic layer with self-protection were installed along with longitudinal gutters among halls.

Another difficult matter was to diminish the visual impact of the air conditioning pipes, unavoidable for the new use of the building ${ }^{40}$. Taking Cabrero, Ruiz and Labiano's guidelines as a reference in the Crystal Pavilion, galvanised steel pipes were installed to the fullest order. Finally, as for the interior decoration, the original concrete benches were recovered.

It was noticed on site that the premanufactured glass capsules altered the Pavilion aesthetics as they were not easily reversible. They were made on site with a minimum $60.60 .2 \mathrm{~mm}$ aluminium tube, perimetral beams and UPN $100 \mathrm{~mm}$ joists, all of them lacquered in red, with aluminium windows and big $5+5 \mathrm{~mm}$ laminar glass panes emulating the logics and aesthetics of the "pavilions-inside-a-pavilion" in order not to interfere with the view among halls ${ }^{41}$ (fig. 12 and 14).

There was a usual problem in modern architecture rehabilitation: minimizing the thermal bridges caused by the p.122 exposed steel structure. This was solved by installing an inner rigid isolating red painted pannel on the core of the beams, hardly perceptible. The glass panes, rebuilt42 as in the pictures, maintain the original partitions changing the structure of steel and aluminium framework which would cause serious condensation problems (see fig. 7 and 14). It was decided to adapt a manufactured framework of $180 \times 60 \mathrm{~mm}$ steel pane lacquered in steel natural tone with thermal bridge breaking and isolation-chamber security glass ${ }^{43}$. Due to the enormous weight of the glasses and their easy replacement, metric 10 steel wires among panes were installed to avoid bending, equalizing the horizontal and vertical frames in the wall, cutting down gaps up to three 1,60 × 2,50cm glasses per gap.

It was used current furniture in black and white colors that would fit the 60s aesthetics and fulfill ergonomic and occupational health needs. Reproducing the original black cube lamps that hung from the structure was an interesting contribution so it would minimize the vision of the air conditioning pipes. The stairs were reproduced keeping the original aesthetics but adapted to the Building Technical Code requirements (fig. 15).

Recognising, protecting and intervening in $20^{\text {th }}$ century architectures has become a regular matter, although we still remain in a context of permanent threat, so there is a long way to go. Intervening symbolic works as the Bauhaus building in Dessau (1925), Ville Savoye in Poissy (1929), the remodelling of the German Pavilion in Barcelona (1929) or more recently, the Gobierno Civil building in Tarragona (1957) we verify the fact that, from agreed general guidelines 
-different from historical heritage preservation guidelines because of the experimental and temporary nature of these architectures- the praxis means a constant review due to, among other factors, the use the building is meant for.

The aim of the works in buildings as the Pavilion in Barcelona or Ville Savoye is that their archeological recovery emulates, not without debate, the spirit of the times they were built in. Talking about Ville la Savoye we could ask ourselves: Is it still the same residence, after so many interventions that keep the building in an everlasting present, not allowing it to become old? ${ }^{44}$ Other buildings preserve their original use, as the Bauhaus building in Dessau or the Gobierno Civil. They try to recover the most of it but with different technics from the original. The Bauhaus building is a subject of intervention considering the "derestoration" in previous stages" 45.

Restorations at the buildings in IIT campus are referential. Crown Hall (1956) is still a College for Architecture 65 years later. The restoration of the curtain walls by Krueck + Sexton ${ }^{46}$ starts by considering pathologies and identifying the causes of corrosion and glass breaks. The original $6 \mathrm{~mm}$ glass panes were replaced by $9 \mathrm{~mm}$ security glass after sandblasting and repainting the structure in its original black colour. The rectangular-section flashing was replaced by trapezoidal flashing in order to displace water and avoid corrosion, without altering the original aesthetics but keeping the thermal bridge all in omitting the energy-efficiency guidelines to preserve Mies's detailed design. The interventions in the campus chapel (1952) are more modest, as the building itself. They consist of cleaning up and renewing the water-proof roof and the redesign of the cornice detail and the replacement of damaged bricks for similar in kind and time bricks from Baily Hall building ${ }^{47}$.

The followed guidelines in the latter buildings are generally based on trying a "rehabilitation in origin" 48 by reproducing the original technics and without complying the current building regulations.

From another point of view, the architectures in the studied case have been expanded by considering modern architecture as a recycling matter, where reuse is a primary matter, not only restoration itself ${ }^{49}$. The proposal found in the campus try to pass on citizenship 20 th century ${ }^{50}$ values and heritage identity by a sustainable building, not as a

p.124 rigid restoration but as a useful rehabilitation. It's a new use respecting the original values, that's why we consider it as a result of an expanded architecture. The main intervention guidelines, according to the Madrid document ${ }^{51}$, have been implemented following each building peculiarities. Two of Cabrero's buildings, Obra Sindical Pavilion in collaboration with Pérez Enciso and the Ministerio de la Vivienda with Ruiz, designed in a three-year gap but so architecturally different have implemented air conditioning or glass railings to fulfill the Spanish Technical Code requirements as well as the industrial parquet on the floors in a homogeneus way following similar intervention guidelines in both buildings.

The campus proposal innovative as it inserts a new socially demanded use in a former exhibition hall but it lacks a strategic plan to coordinate this kind of intervention. Obviously, the exhibiting use remains primary. That's what the pavilions were meant for. The value of this modern architecture and the need to preserve it is out of question. Their reversible recycling as a second opportunity with the use according to the Plan does not prevent other pavilions to be rehabilitated as the Hexágonos or the Convention Center, both exhibition halls as the Crystal Pavilion, adding value to the enclosure activity.

As far as it concerns the interventions in the Exhibition Pavilion, it's a matter of solving the difficult equation of preserving a non-registered building of great architectural and cultural value, lacking original elements and poorly planned with serious pathologies due to the economic situation and the ephemeral nature of the Fairgrounds. In fact, the only preserved and recovered original element is the terraced concrete structure and steel frameworks. The survival of the main element in the "iron age" is food for thought as the rest of the elements consist of a "dressing" to this main element that shapes the space. Cabrero would probably be satisfied. Unprecedented pictures of the enclosing free structure are an expression of modernity, of "social-utilitary aim" as well as an "expression of beauty" that should go along with architecture, either in origin or expanded architecture.

This article outlines the results of a Research Project lead by the author Ref: 43824812045 "Asistencia Técnica para el pabellón de Exposiciones" within the LOU 83 article, undersigned by Fundación General de la Universidad Politécnica de Madrid and Ferrovial Construcción S. A. financial corporation.■

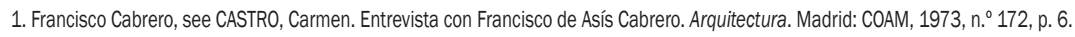

2. See: COCA, José de. El recinto ferial de la Casa de Campo de Madrid (1950-1975). Directores: José María Mercé y Javier Ortega. Tesis doctoral. Universidad Politécnica de Madrid, Departamento de Proyectos Arquitectónicos, 2013.

3. Ibídem, p. 22. Made by the architect Luis Labiano. The 123 buildings were valued at 2,059,800,000 pesetas.

4. Preliminary Studies in Plan Especial "Feria del Campo" AOE 00.04. Vol. 1 Memoria. Ayuntamiento de Madrid, 2002.

5. IFEMA archives. General Urban Planning Information Recinto Ferial de la Casa de Campo Madrid. Madrid City Hall, December 1984. Javier de la Calzada and Juan Carlos García Barbería, architects.

6. The tragic accident where five teenagers were killed on the $1^{\text {st }}$ of November 2012.

7. Special Plan about the use and works in protected buildings belonging to Casa de Campo Fairgrounds. Madrid City Hall: Financial, Economic and Public Administration Department, 2014 [consulted 29-09-2020]. Available at: ftp://rfa.munimadrid.es/PContratante/publicados/0140e2dba4645410VgnVCM1000000b205a0aRC RD/1397117287839/CIUDAD_SEGURIDAD_02.pdf.

8. The Special Plan "Feria del Campo" AOE.00.04 is established in 2004 and definitively approved in 2006. The catalogue was carried out by José de Coca Architects under the guidance of J. Gago and J. M. García-Pablos. The Plan develops the policies submitted by the 1997 PGOUM. Highly commended at Architecture, Urban Planning and Public Works 2006 Madrid City Awards. 
9. Special Plan "Feria del Campo" AOE.00.04. Madrid: Ayuntamiento de Madrid, 2006.

10. Ibíd. Appendix 1: Catalogue of Buildings and Architectural Elements.

11. COCA, José de, op. cit. supra, nota 2, p. 268. Unfortunately, even been protected, they have been removed and replaced by the "Fernandinas" traditional model.

12. Campus Asociativo de Madrid Dossier. Madrid: Spatial Planning and public-private sector Cooperation, 2018 [consulted 29-09-2020]. Available at: https://diario.madrid. es/wp-content/uploads/2018/12/Dosier-Campus-Asociativo-de-Madrid.pdf

13. Between June 2015 - 2019 ruling Mayor Manuela Carmena.

14. Campus Asociativo Dossier, op. cit. supra, nota 13, pp. 13-14.

15. MATA, Sara de la; SOBEJANO, Enrique. Entrevista Francisco de Asís Cabrero. In: Arquitectura. Madrid: COAM, 1987, n. ${ }^{\circ}$ 267, pp. 110-115.

16. See CABRERO, Francisco de Asís. Las tres naturalezas del neoplasticismo. In: The four books of Architecture. Book III. Madrid: COAM, 1992, pp. $342-343$.

17. "La retícula y el hierro", see: RUIZ CABRERO, Gabriel. Vida y obra de Asís Cabrero. In: AAVV, Francisco de Asís Cabrero. Legado 02. Madrid: Fundación COAM, 2007, p. 67. 18. CABRERO, Francisco de Asís. Francisco de Asís Cabrero. Título 1942. In: Arquitectura. Madrid: COAM, 1964, n. ${ }^{\circ}$ 61, p. 16.

19. CASTRO, op. cit. supra, note 1, p. 6 .

20. SÁNCHEZ DE LA CHICA, Juan Manuel. Materia, material y aparejo en la arquitectura de Francisco de Asís Cabrero. Director: Gabriel Ruiz Cabrero. PhD tesis, ETS de Arquitectura Universidad de Málaga, 2015, p. 342.

21. See: "arquitectura desornamentada". CABRERO, op. cit. supra, note 16, p. 60.

22. CASTRO, op. cit. supra, note 1, p. 5.

23. Íbid, p. 6.

24. Pabellón del Ministerio de la Vivienda, IV Feria Internacional del Campo. Casa de Campo Madrid. Legado 02 Francisco de Asís Cabrero. Fundación COAM, 2007. FCT/ P104, FCT P449.

25. HARBOE, Gunny. Restoring the "God Box": Mies van der Rohe's Carr Chapel at IIT. Ludwig MIES VAN DER ROHE et al. The Heritage of Mies. Eindhoven: Eindhoven Docomomo International Secretariat, 2017, n. ${ }^{0} 56$ (2017/1), p. $73-77$.

26. In Cabrero's archives, there is only the numbered register (plans 2791 to 3840). Not even a summary was preserved, just some photographs. See COCA, José de, op. cit. supra, note 2, p. 315.

27. Measured in situ to pilars. Both central halls present $11.52 \mathrm{~m}$ long and $11.50 \mathrm{~m}$ wide stretches. At the entrance hall, they are $11.36 \mathrm{~m}$ and $11.82 \mathrm{~m}$. At the lower hall, stretches are $11.28 \mathrm{~m}$ and $11.35 \mathrm{~m}$. This adjustment from the $11.50 \mathrm{~m}$ model respond to the use of the space and the relative position among pilars and beams due to the "fullest" joinings.

28. CLIMENT, Javier. Francisco Cabrero, arquitecto: 1939-1978. Madrid: Xarait, 1979, p. 96.

29. Pabellón del Ministerio de la Vivienda. Arquitectura. Madrid: COAM, julio 1959, n. ${ }^{\circ}$ 7, pp. 16-19.

30. Spatial solution similar to the residence in Miraflores (1957) by Alejandro de la Sota, R. V. Molezún and J. A. Corrales, unrecognisable nowadays.

31. Particular uses: fairs, cultural and sports events, catering and education. Temporarily accepted: security, civil defence, courts or similar.

32. Operative programme of Complejo Municipal de Impulso del Asociacionismo, 7 th of December 2017.

33. Buildings Catalogue, Ficha n. ${ }^{\circ}$ 41. Plan Especial "Feria del Campo" AOE 00 04. Madrid: Ayuntamiento de Madrid, 2006.

34. Rehabilitation Project of buildings managed by Madrid Destino in Casa de Campo Fairgrounds. Complejo Municipal de Impulso del Asociacionismo. Ed. Pabellón de Exposiciones. INCOSA S. A. Arquitecto: David Espigado. Madrid, noviembre de 2018.

35. The big ones measuring $3.45 \times 9.9 \mathrm{~m}$; the small ones $3.45 \times 6 \mathrm{~m}$ transversal and $2.70 \mathrm{~m}$ high.

36. According to the rehabilitation Project, the upper limitation states reach $98 \%$ of the acceptable tension. Maximum deflection due to the global load is $105 \mathrm{~mm}$, exceeding the admissible limitation (L/250) but they do not endanger the structure security, which has perfectly lasted for 60 years. The roof lightening allows the overload and reduces the permanente deflection.

37. Testimony of Jaime Ruiz. See COCA, José de, op. cit. supra, note 2, p. 74

38. Technical Assistance Agreement undersigned by the Fundación General de la Universidad Politécnica and Ferrovial Agromán S. A. contracted company. It would have been desirable to assign the assistance already during the project drafting.

39. RAL CLASIC 3011 "brownish-red”. RGB code Red: 121 (47 \%), green: 36 (14 \%), blue: 35 (14\%).

40. It was considered to earth the pipes up and use the brick chambers as impulse and return channels. An ingenious solution but economically unfesiable and technically complex. 41. They were installed on site with an individual air-exchange unit in the hall.

42. As there are no plans or original elements, the recovery of the building detail is sheer hypothesis. See MARTíNEZ DÍAZ, Ángel; ORTEGA VIDAL, Javier. Investigación y reconstrucción gráfica. In: Actas 13 Congreso Internacional de Expresión Gráfica Arquitectónica. Valencia: Universitat Politécnica de Valencia, 2010, pp. 281-286.

43. Aluminium-tone lacquered zinc-steel curtain wall with EPDM junctures and outdoor stainless-steel jambs. Double $8+8.2 / 16 \mathrm{Ar} / 6+6.2$ glass. Annealed glass with 2 butiral layers, solar control and low emission layer, argon 16mm chamber and double perimetral butil-sillicon-UV seal. Each glass pane weighs $280 \mathrm{~kg}$.

44. MARTÍNEZ MEDINA, Andrés. Las huellas del tiempo de la arquitectura moderna intervenida. In: AAVV. Criterios de intervención en el patrimonio arquitectónico del siglo XX. Conferencia Internacional CAH20thC. Documento de Madrid 2011. Madrid: Ministerio de Cultura, 2011, pp. 395-401.

45. See MARKRAF, Monika; OELKER; Simone; SCHWARTING, Andreas. Denkmalplege der Moderne. Konzepte für ein junges Architekturerbe. Wüstenrot Stiftung. Stuttgart: Karl Krämer Verlag, 2011.

46. SEXTON, Mark. Restoration of Crown Hall. En: Ludwig MIES VAN DER ROHE et al., op. cit. supra, nota 25, pp. 65-71.

47. HARBOE, G., op. cit. supra, nota 25, pp. 73-77.

48. MARTÍNEZ MEDINA, Andrés, op. cit. supra, nota 44, p. 398.

49. MARTÍNEZ MONEDERO, Miguel. Reciclaje de arquitecturas vs. restauración arquitectónica, ¿̇herramientas contrapuestas? En: Hábitat y Sociedad. Reciclaje de barrios [en línea]. Sevilla: Universidad de Sevilla, 2012, n. ${ }^{\circ}$ 5, pp. 23-33 [consulted: 17-02-2021]. ISSN: 2173-125X Disponible en: https://revistascientificas.us.es/index.php/HyS/ article/view/4131 Dol: https://doi.org/10.12795/HabitatySociedad.2012.15.03

50. Art. 9.2 “Comunicar los valores del patrimonio de forma amplia”. En: AAV. Criterios de intervención en el patrimonio arquitectónico del siglo XX, op. cit. supra, nota 44, pp. 23-28.

51. Ídem. 

CITY. Darío Álvarez Álvarez • LINAZASORO EN REIMS. EL ESPACIO PÚBLICO COMO MEMORIA DEL LUGAR / LINAZASORO IN REIMS. THE PUBLIC SPACE AS THE MEMORY OF THE PLACE. Victoriano Sainz Gutiérrez • EDITAR VS. CONSTRUIR: UNA ECOLOGÍA DE LO INVISIBLE. AMPLIFICAR LA COMPRENSIÓN DE LAS TÉCNICAS DE PROYECTO / EDITING VS. BUILDING: AN ECOLOGY OF THE INVISIBLE. AMPLIFYING THE UNDERSTANDING OF ARCHITECTURAL DESIGN TECHNIQUES. Paula Victoria Álvarez Benítez • CUANDO LA PINTURA AMPLÍA LA ARQUITECTURA: INTERVENCIONES REALIZADAS EN EL ESPACIO PÚBLICO / WHEN PAINTING ENHANCES ARCHITECTURE: INTERVENTIONS IN A PUBLIC SETTING. Aurora Alcaide-Ramírez; Ana Ruiz-Abellón • UN EDIFICIO INVISIBLE. NUEVO AULARIO DE LA FACULTAD DE DERECHO DE LA UNIVERSIDAD DE ZARAGOZA (1983-1996) / AN INVISIBLE BUILDING. NEW LECTURE ROOM BUILDING OF THE FACULTY OF LAW OF THE UNIVERSITY OF ZARAGOZA (1983-1996). Luis Miguel Lus-Arana; Lucía Carmen Pérez-Moreno • ARQUITECTURAS AMPLIADAS. EL PABELLÓN DE EXPOSICIONES EN LA CASA DE CAMPO DE MADRID) / EXPANDED ARCHITECTURES. THE EXHIBITION PAVILION AT THE CASA DE CAMPO IN MADRID. José de Coca Leicher $\bullet$

- RAFAEL MONEO VALLÉS: LA VIDA DE LOS EDIFICIOS. LA MEZQUITA DE CÓRDOBA, LA LONJA DE SEVILLA Y UN CARMEN EN GRANADA . Víctor Pérez Escolano • FRANCISCO DE GRACIA: CONSTRUIR EN LO CONSTRUIDO. LA ARQUITECTURA COMO MODIFICACIÓN. Pablo Diañez Rubio • FRÉDÉRIC DRUOT, ANNE LACATON \& JEAN-PHILIPPE VASSAL PLUS: LA VIVIENDA COLECTIVA. TERRITORIO DE EXCEPCIÓN. Javier Terrados Cepeda.
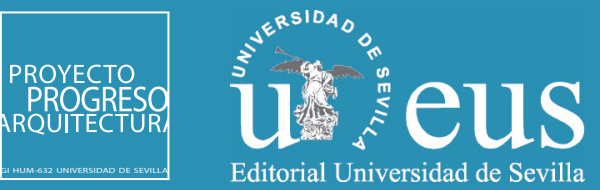